\title{
Analysis of Membrane Deploying Process Based on Miura Origami
}

Jing Zhang ( $\square$ free1985216@163.com )

Taiyuan University of Technology https://orcid.org/0000-0001-5735-8132

\section{Youqing Shen}

Taiyuan University of Technology

\section{Hongwei Guo}

Harbin Institute of Technology

\section{Rongqiang Liu}

Harbin Institute of Technology

\section{Ziming Kou}

Taiyuan University of Technology

\section{Original Article}

Keywords: Membrane structure, Crease length, Number of elements, Longitudinal crease angle, Deployment synchronization

Posted Date: September 28th, 2021

DOl: https://doi.org/10.21203/rs.3.rs-917429/v1

License: (c) (i) This work is licensed under a Creative Commons Attribution 4.0 International License. Read Full License 


\section{Abstract}

Aiming at a series of characteristics of the membrane structure in the folded configuration during the deploying process, the initial defect of the membrane crease is introduced by proxy model, and to realize the study of the deploying process of the membrane. Based on the finite element method, the deploying process of the multi-element Miura membrane is simulated. And the stretch ratio, maximum mises stress, deployment rate, wrinkle deformation, etc. of membrane structure are discussed. The existence of creases can cause damage to the membrane surface, so the total length of the creases should be within an appropriate size range. By changing the number of elements and the longitudinal crease angle of the same size membrane respectively, the influence of the above two factors on the total crease length, storage volume and deploying process of the folded membrane is studied. The results show that when the longitudinal crease angle is $15^{\circ}$, the transverse and longitudinal displacements of the Miura folded membrane with different element numbers are not synchronized during the deploying process. By keeping the number of elements constant and increasing the angle of the longitudinal creases from $15^{\circ}$ to $45^{\circ}$, the synchronization of the transverse and longitudinal displacements during the membrane deploying process is gradually enhanced. In addition, the experiment on the membrane deploying process verifies the reliability of the finite element simulation results.

\section{Introduction}

When the membrane structure performs normal space tasks, the effective area of its reflective surface can reach $50.265 \mathrm{~m}^{2} .{ }^{1}$ Therefore, the membrane structure will be folded or rolled up during the launching process of the rocket and deployed on the orbit to reach the working state. Thereby reducing the transportation cost and task difficulty of future space missions. ${ }^{2}$ During the folding process, the membrane exists elastic-plastic deformation, resulting in creases. ${ }^{3}$ The existence of creases changes the state and material behavior of the membrane surface. And it has a certain degree of irreversibility when the membrane is fully deployed, ${ }^{4,5}$ and has a certain degree of influence on the deploying process and deployment results of the folded membrane. In addition, when the arrangement of the creases is changed to enhance or weaken the correlation between the creases, the membrane can also have different deployment behaviors during the deploying process.

The creases of the Z-fold membrane are parallel to each other, and the configuration is relatively simple, so it has become one of the hotspots for studying the deployment behaviors of the membrane.

Mierunalan et al. used the finite element method to study the Z-folding and free-rebound processes of the membrane, and studied the influence of the material type, folding degree and membrane thickness on the release configuration of the membrane. The simulation results corresponded well with the experimental results. ${ }^{6}$ Satou and Furuya et al. studied the elastic-plastic behavior of the crease, simulated the free rebound of the membrane and the subsequent deploying process, and discussed the influence of the membrane thickness on its deploying process. The final numerical analysis results are consistent with the experimental results. ${ }^{7}$ Xia et al. proposed a crease-beam model, which considers the bending and 
crease expansion of the membrane to describe the deployment behavior of the Z-folded membrane under tensile load. ${ }^{8}$

Since the complex crease pattern plays an important role in the realization of small membrane folding volume. Therefore, it is of great significance to study the deploying process of membranes with complex crease patterns. ${ }^{9}$ Papa et al. conducted a deployment experiment on a square membrane packaged in the Miura Origami, and studied the load-displacement response of the membrane during the deploying process. ${ }^{10} \mathrm{Xu}$ Yan et al. used the spring-mass system to simulate the deploying process of the outer leaf folding, the inner leaf folding and the Miura folding respectively, and studied the deployment stability of the above three folding methods through system kinetic energy and system strain energy. ${ }^{11}$ Arya et al. carried out a deployment experiment on the rotating deployed membrane to studying the influence of the thickness and crease density on the deployment force. ${ }^{12}$ Cai et al. used the effective modulus to simulate the crease of the membrane to realize the deployment simulation of the Miura membrane, and optimized the boundary of the membrane to reduce the stress concentration phenomenon of the membrane after deployment. ${ }^{13}$ Shen et al. proposed a proxy model to replace membrane creases. The finite element method was used to simulate the deploying process of Miura membrane, and the effects of different driving modes on the deployment stability of Miura membrane were studied. ${ }^{14}$ However, when the references [13] and [14] researched on the deployment of Miura membrane, the number of parallelogram elements in the Miura pattern was too small. When a large-scale membrane is folded, it will produce multi-element crease patterns, so it is of great significance to study its deploying process.

In this paper, the proxy model in the reference [14] was used to replace the creases to introduce initial defects into the membrane to realize the study of the membrane deploying process. The finite element method was used to simulate the deploying process of the multi-element Miura membrane. The influence of the number of element and the longitudinal creases angle on the total length of the crease, the storage volume, and the synchronization of the transverse and longitudinal bidirectional displacements during the membrane deploying process were studied. Finally, experiments were used to verify the reliability of the finite element simulation results.

\section{Membrane Folding And Deployment Analysis}

In this section, the deployment simulation of the multi-element Miura membrane was carried out, in which the membrane crease was replaced by proxy model. A series of characteristics such as deployment rate, deployment error, maximum Mises stress, stretch ratio and wrinkle deformation were studied.

\subsection{Membrane material and crease proxy model}

PET (Polyethylene terephthalate) membrane has excellent mechanical properties with tensile strength and impact strength higher than ordinary membranes. And it is used more and more frequently in space tasks. The PET membrane is used for the study, with thickness of $100 \mu \mathrm{m}$, yield stress of $65 \mathrm{MPa}$, and Young's modulus of about $4800 \mathrm{MPa} .{ }^{15}$ 
The proxy model is used to replace membrane creases. ${ }^{14}$ This method mainly uses ABAQUS Hinge connectors to connect the membrane mesh nodes to replace the crease connections between the membrane surfaces. The crease width is defined based on the deploying simulation of Z-fold membrane, which was given in reference [14]. The ABAQUS connector behavior simulates the elastic-plastic behaviors of the membrane creases. The connection method of the ABAQUS connector is shown in Fig. 1, and the elastic-plastic behavior at the crease is shown in Fig. 2. The same method is used to analyze the folding and deployment of the multi-element Miura membrane, in which the behavior at the crease is simulated only by the nonlinear elastic behavior of the ABAQUS connector.

\subsection{Modeling of Miura membrane}

The $210 \mathrm{~mm} \times 210 \mathrm{~mm}$ square PET membrane is used as the research object, and the Miura Origami method is used. The number of parallelogram elements is $5 \times 5$, as shown in Fig. 3 . It is worth noting that the solid red line is the boundary of the membrane, and the part of the parallelogram element that exceeds the boundary will be removed. The dotted line creases are valley creases, the solid line creases are mountain creases. And the angle between the longitudinal crease and the $y$-axis direction is $15^{\circ}$. The ABAQUS connector is used to connect the mesh nodes in the membrane crease area, and the elasticplastic behavior of the connector replaces the elastic-plastic properties of the crease. In addition, in order to avoid excessive stress concentration at the four corners of the membrane when the deployment force is too large, the four corners are removed and replaced by a linear loadable region that is $45^{\circ}$ or $135^{\circ}$ with the positive $x$-axis. The side length of this linear loadable region is $10 \mathrm{~mm}$, as shown in the partial enlarged area in Fig. 3.

\subsection{Analysis of membrane folding and deploying process}

\subsubsection{The folding and deploying process of membrane}

Taking the fully folded membrane as the research object, the deploying process of the membrane is discussed. It is known that the membrane still has a certain elastic deformation at the crease after it is fully folded, so the membrane will have a certain degree of free rebound after being relaxed. Assuming that the elastic-plastic properties of each crease of the membrane are consistent, the deployment characteristic curve is shown in Fig. 2. The area in the membrane that is about to produce creases is removed, and the mesh nodes are connected by hinge connectors. The elastic-plastic properties at the creases are used as the connection behavior of hinge connectors, so the proxy model is set up. Using the ABAQUS/Standard solver to perform quasi-static simulation, the membrane in a flat state will gradually folded under the action of the connector's connection behavior, until it reaches a stable state formed by the free rebound of the membrane without a proxy model instead of the crease after it is fully folded. The restriction of the membrane is shown in Fig. 4. Among them, U1, U2, U3 represent the displacement degrees of freedom in the $X, Y$, and $Z$ directions, respectively. And UR1, UR2, and UR3 represent the rotation degrees of freedom in the $X, Y$, and $Z$ directions, respectively. The membrane folding process is shown in Fig. 5 , where the mesh size is $1 \mathrm{~mm}$ and the simulation time is $1 \mathrm{~s}$. 
It can be seen from Fig. 5(e) that when the membrane is in a release configuration, the stress concentration phenomenon mainly occurs at the intersection of the creases, and the maximum Mises stress is $22.41 \mathrm{Mpa}$. In other places, such as the crease area far away from the intersection of the crease, there is no stress concentration phenomenon, and the stress value is small. In this release configuration, a linear force $T$ parallel to the xoy plane is applied to the four linear loadable regions to gradually deploy the membrane. The deployment force $T$ is gradually increased from $0 \mathrm{~N} / \mathrm{mm}$ to $10 \mathrm{~N} / \mathrm{mm}$. The deploying process of membrane is shown in Fig. 6, and the specific application direction of the deployment force $T$ is marked in Fig. 6(a).

\subsubsection{Deployment result of membrane}

(1) The deployment rate and deployment error

During the membrane deploying process, the projected area along the normal direction of the ideal membrane fully deployed plane (the xoy plane in Fig. 6) can be recorded as the effective area. The effective area is an important parameter of the space membrane front structure. Its ratio to the ideal deployment area is defined as the deployment rate of the membrane. ${ }^{14}$

In addition, the neutral surface of the membrane during the deploying process is analyzed, and the two planes parallel to the ideal deployment plane of the membrane are used to encompass the actual deployment neutral surface of the membrane. And the minimum distance between the two parallel planes is regarded as the deployment error. ${ }^{14}$ The deployment error during the membrane deploying process is marked in Fig. 7.

The deployment rate and the deployment error are used to describe the changes of the membrane in the effective area projection plane and the normal direction of the effective area projection plane. Since the simulation is quasi-static, there will be no vibration in the dynamic simulation, and each sample can reflect the equilibrium state of the membrane at this time.

Fig. 8(a) shows the change of the deployment rate during the deploying process of membrane. Since the membrane has elastic deformation at the crease after folded, it will produce free rebound behavior. The effective area of the membrane in the release configuration is $1364.97 \mathrm{~mm}^{2}$, and the deployment rate is $3.1 \%$. Subsequently, the deployment force $T$ is applied to continue the deployment of the membrane. When $0 \mathrm{~N} / \mathrm{mm}<T \leq 0.16 \mathrm{~N} / \mathrm{mm}$, the slope value of the deployment rate curve fluctuates around 3.98 , but its rapid growth trend remains unchanged. When $0.16 \mathrm{~N} / \mathrm{mm}<T \leq 1 \mathrm{~N} / \mathrm{mm}$, the slope value of the deployment rate curve gradually decreases and approaches zero. In this interval, the deployment rate increases to $96.26 \%$ at the end of the curve. And the effective area of the membrane is $42356.56 \mathrm{~mm}^{2}$, which basically meets the application requirements.

When $T=1 \mathrm{~N} / \mathrm{mm}$, although the deployment rate of the membrane is $96.26 \%$, and its effective area is already close to the state when the membrane is deployed, the deployment error is still very large, which is 
$15.68 \mathrm{~mm}$. When the condition of the deployment rate is satisfied, the deployment error during the membrane deploying process is shown in Fig. 8(b).

As shown in Fig. 8(b), the overall curve shows a downward trend, but the rate of decline gradually decreases. When $T=6 \mathrm{~N} / \mathrm{mm}$, the deployment error of the membrane is $1.79 \mathrm{~mm}$. Since the deployment error when the membrane fully folded is $42 \mathrm{~mm}$, the solution error of the deployment error at this moment is $4.26 \%$. In addition, when $6 \mathrm{~N} / \mathrm{mm}<T \leq 10 \mathrm{~N} / \mathrm{mm}$, the rate of reduction of the deployment error approaches zero. At the end of the curve, the deployment error is $1.25 \mathrm{~mm}$, and the average value of the longitudinal crease deployment angle is $69.94^{\circ}$, the average value of the transverse crease deployment angle is $71.37^{\circ}$. The rotation angle of the connector is still within the effective range of the proxy model instead of the membrane crease. Due to the elastic deformation of the membrane, although the creases have not yet reached the fully deployed state, the deployment rate of the membrane has reached $100 \%$.

In summary, a certain degree of deployment force can make the membrane deployment rate reach the condition that the solution error is less than $5 \%$, but if the solution error of the membrane deployment error is less than $5 \%$, the above-mentioned deployment force needs to be increased to $5-6$ times of its own.

(2) In-plane stretch ratio

The deployable structure must have a large enough size to meet the working requirements after deployment, but it still need to meet the storage requirements of small volume after folding. Therefore, it needs to be calculated for the stretch ratio. Considering that the effective area is an important parameter of the space membrane front structure, the ratio of the effective area of the membrane in the deployed configuration to the release configuration is taken as its in-plane stretch ratio. Obviously, when the membrane conforms to the small-volume folding, its in-plane stretch ratio is very important. ${ }^{16}$

$$
\eta=S_{1} / S_{0}
$$

In the formula, $S_{1}$ is the effective area after the membrane structure is deployed, and $S_{0}$ is the effective area when the membrane structure is released. The in-plane stretch ratio of the $5 \times 5$ elements membrane is 32.24 , and the envelope volume in release configuration is $102.64 \mathrm{~cm}^{3}$.

(3) Mises stress

It can be seen from the membrane deploying process in Fig. 6 . When $T \leq 1 \mathrm{~N} / \mathrm{mm}$, although the four linear loadable regions of the membrane have the effect of deployment force $T$, the stress concentration area mainly exists the membrane crease area. With the gradual increase in the deployment force $T$, the maximum Mises stress point gradually approaches the intersection of the creases. When $1 \mathrm{~N} / \mathrm{mm}<T \leq 10 \mathrm{~N} / \mathrm{mm}$, the stress concentration phenomenon in linear loadable regions of the membrane gradually intensifies. At the end of the deployment force growth, the maximum Mises stress exists in linear loadable regions, which is $91.98 \mathrm{MPa}$. 
(4) Wrinkling phenomenon of deployed membrane

The thickness of the membrane structure is on the order of micrometers, its bending stiffness is very small. And it is prone to local instability when subjected to force, and then wrinkles. ${ }^{17}$ Affected by wrinkles, the membrane surface precision is reduced, and the working performance of the lightweight front reflection structure made of membrane materials is greatly reduced. There are two main types of wrinkles in membrane structure: one is material wrinkles, also called plastic wrinkles, which are irreversible wrinkles caused by plastic deformation of membrane materials during processing or in the folded configuration; the other is structural wrinkles, also called elastic wrinkles, it is a kind of buckling response behavior of the membrane under the action of external force. The structural wrinkle is completely reversible, and its amplitude changes with the force state. ${ }^{18}$

In this paper, the membrane is folded, plastic deformation occurs at the crease, so there are material wrinkles. In addition, after the membrane is deployed, wrinkles appear on the surface of the non-crease area, so there are structural wrinkles. In the case of deployment force $T=10 \mathrm{~N} / \mathrm{mm}$, the displacement of the $1 / 4$ part of the membrane in the Z-axis direction is shown in Fig. 9.

Since the square membrane has a symmetrical structure, the material wrinkle of the structure is generally symmetrical about the symmetry axis shown in the Fig. 9. However, since the creases are asymmetric, the wrinkle shape of the membrane after the overlap of the material wrinkles and structural wrinkles is asymmetric. The A-A path passes through the intersection of the crease and is parallel to the membrane linear loadable region with distance of $48.9 \mathrm{~mm}$. Since the wrinkle shape of the membrane that changes along this path is more obvious, the coordinate axis is established from the bottom left to the top right as the positive direction, and the starting point of the membrane surface on the A-A path is used as the coordinate axis origin to analyze the out-of-plane deformation (the deformation in the Z-axis direction) of the membrane. The specific value of the deformation is shown in Fig. 10.

It can be seen from the Fig. 10 that the curve has three local maximum points and two local minimum points. Considering the accumulation of material wrinkles caused by many creases, the out-of-plane deformation of the membrane along the A-A path is all negative. The second local minimum point has the largest Z-axis negative out-of-plane deformation, which is $1.18 \mathrm{~mm}$. And the third local maximum point has the smallest Z-axis negative out-of-plane deformation, which is $0.23 \mathrm{~mm}$. Among them, the position of the largest negative out-of-plane deformation of the Z-axis is the position of the intersection of the creases, where has the largest amount of material wrinkles along the A-A path. Together with the effect of structural wrinkles, the current out-of-plane deformation of the membrane is formed.

In addition, the out-of-plane deformation curve of the membrane near the two end points of the A-A path does not fluctuate up and down and converges to a certain stable value. This is because the end points on both sides are close to the mountain crease area of the membrane, which causes the out-of-plane deformation near the end points on both sides showed a tendency to increase from the inside to the edge of the membrane. 


\section{The Influence Of The Crease Layout On The Folding And Deploying Process Of The Membrane}

\subsection{Influence of the number of parallelogram elements}

The appearance of creases not only limits the accuracy of the membrane structure, but also damages the membrane to a certain extent, which significantly affects the shape and performance of the membrane structure. It is one of the classic failure modes of membrane deployable structures that require high precision. Therefore, when the membrane is fully folded, the total length of the creases should be as short as possible while meeting the requirements of the storage volume. Membranes of the same size with parallelogram elements of $3 \times 3,5 \times 5$, and $7 \times 7$ are folded, and the difference in the envelope volume is discussed. In addition, when the total length of the crease changes, the membrane will have different deployment behavior under the same deployment force. The deployment force is applied to the folded membranes of the above three types of crease division methods to observe the difference in the deploying process.

Fig. 11 shows the division of the creases of the membrane by the number of parallelogram elements of $3 \times 3,5 \times 5$, and $7 \times 7$. The comparison of the folded storage volume and the total length of the creases with different numbers of parallelograms is shown in Table 1.

Table 1 The folded membrane parameters.

\begin{tabular}{llll} 
Number of elements & $3 \times 3$ & $5 \times 5$ & $7 \times 7$ \\
\hline Length of crease $(\mathrm{mm})$ & 854.82 & 1709.64 & 2564.46 \\
\hline Deployment error $(\mathrm{mm})$ & 69.65 & 42.73 & 29.85 \\
\hline Envelope volume $\left(\mathrm{mm}^{3}\right)$ & 203979.78 & 102641.97 & 52068.67
\end{tabular}

It can be seen from Table 1 that with the increase of the number of parallelograms, the total length of the crease increases exponentially, and the damage to the membrane surface becomes more serious. Although the structural wrinkles amplitude of the deployed membrane will not increase significantly, the density of structural wrinkles will increase. In severe cases, the membrane surface will appear bumpy, so that the membrane cannot work as expected effect. The deployment error of the membrane in the folded configuration is affected by the size of the parallelogram element, and it increases with the increase of the size of the parallelogram element. Therefore, with the increase of the number of parallelograms, the envelope volume of the folded membrane decreases obviously.

The folded configuration membranes with the number of parallelogram elements of $3 \times 3,5 \times 5$, and $7 \times 7$ is respectively applied with deployment force. Fig. 12 shows the intermediate state of the deploying process of the $7 \times 7$ membrane. Due to the elastic deformation of the membrane parallelogram element, the displacement changes at the four corners of the membrane cannot truly reflect the deployment of the overall membrane structure. Therefore, compared to discussing the displacement of the linear loadable 
region, discussing the displacement of the relatively centered transverse and longitudinal bidirectional creases on the overall membrane surface in their respective directions is more suitable for the overall deployment movement of the membrane structure.

According to the Fig. 12, $\left|a a_{1}\right|_{x}$ and $\left|b_{1}\right|_{x}$ are defined to respectively represent the length of the two transverse creases in the $x$ direction, and use the average value of the two as the deployment distance of the membrane in the $x$ direction, which is denoted as $I_{x} \cdot \mid c_{1} l_{y}$ and $\mid d_{d} d_{1}$ respectively represent the length of the two longitudinal creases in the $y$ direction in the figure, and the average value of the two as the deployment distance of the membrane in the $y$ direction, which is recorded as $l_{\mathrm{y}}$. During the membrane deploying process, the Ramp amplitude curve is defined for the deployment force $T$, which means a linear transition between the initial state and the end state of the entire analysis step. Among them, the deployment time is $10 \mathrm{~s}$ in total, and the deployment force $T$ peak value is $1 \mathrm{~N} / \mathrm{mm}$.

The changes of $I_{x}$ and $l_{y}$ with the deployment force $T$ are shown in Fig. 13 (a) and Fig. 13 (b). With the gradual increase of the deployment force, the transverse and longitudinal bidirectional displacement of the membrane of the multi-parallelogram element shows an increasing trend, and the transverse deploying progress has a certain lag relative to the longitudinal. The longitudinal creases in the membrane surface deploy first and the deployment rate is high. Although the deployment rate of the transverse creases gradually increases, the deployment rate of the transverse creases is still low when the longitudinal creases are not fully deployed. When the longitudinal crease is nearly fully deployed, the transverse crease deploys quickly so that the effective area of the

membrane meets the working size requirements. It is worth noting that changing the number of parallelogram elements of the same size membrane will result in a different total length of membrane creases. The greater the number of parallelogram elements, the greater the total length of the membrane creases, the smaller the growth rate of the transverse displacement $I_{x}$ and the longitudinal displacement $l_{y}$, and the longer the deployment time. However, all membranes can reach a fully deployed configuration by increasing the deployment force.

In addition, the required deployment force of the folded membrane increases exponentially in the case of the same transverse displacement $I_{x}$ or longitudinal displacement $I_{y}$ with the number of parallelogram elements of the same size membrane increases. Although at the end of the curve, that is, when the deployment force $T=1 \mathrm{~N} / \mathrm{mm}$, the transverse and longitudinal displacement of the membrane has approached the fully deployed value, but the deployment angle at the membrane crease at this moment is still very small, and the deployment error is still large. Although the membrane can be fully deployed by continuing to increase the deployment force $T$, the increase in the transverse and longitudinal displacement of the membrane is very limited.

\subsection{Influence of the longitudinal crease angle}


In addition to the number of parallelogram elements, the longitudinal crease angle can also affect the total length of the membrane crease. The folded membrane with a parallelogram element of $5 \times 5$ is used as the research object. By changing the angle of the longitudinal crease, the difference in the envelope volume and the deploying process is studied.

The division of the membrane by longitudinal creases with the crease angles of $15^{\circ}, 30^{\circ}$, and $45^{\circ}$ is shown in Fig. 14. The comparison of the envelope volume and the total length of the creases of the membrane with different longitudinal crease angles is shown in Table 2.

Table 2 The folded membrane parameters.

\begin{tabular}{llll} 
Longitudinal crease angles $\left(^{\circ}\right)$ & 15 & 30 & 45 \\
\hline Length of crease $(\mathrm{mm})$ & 1709.64 & 1809.95 & 2027.94 \\
\hline Deployment error $(\mathrm{mm})$ & 42.73 & 40.33 & 36.12 \\
\hline Transverse displacement $(\mathrm{mm})$ & 95.02 & 143.87 & 175.65 \\
\hline Longitudinal displacement $(\mathrm{mm})$ & 25.28 & 23.99 & 22.10 \\
\hline Envelope volume $\left(\mathrm{mm}^{3}\right)$ & 102641.97 & 139196.63 & 140212.96
\end{tabular}

It can be seen from Table 2 that with the increase of the longitudinal crease angle, the total length of the membrane creases increases, and the damage to the membrane surface gradually becomes serious. Different from increasing the number of parallelogram elements, changing the angle of the longitudinal creases does not change the total number of creases. The change in crease length is only caused by the layout of the creases, so the increase in the total length of the creases is limited. In addition, the deployment error and the longitudinal displacement of the membrane folded configuration decrease with the increase of the longitudinal crease angle. But the transverse displacement of the membrane folded configuration is significantly increased. The change in the transverse displacement of the membrane folded configuration is mainly caused by the angle change of the longitudinal crease, which is a design problem of the crease layout. In general, the envelope volume of the membrane tends to increase due to its transverse displacement with the increase in the angle of the longitudinal creases.

Folded membranes with longitudinal crease angle of $15^{\circ}, 30^{\circ}$, and $45^{\circ}$ are respectively applied with deployment force to achieve deployed, and the displacement of the relatively centered transverse and longitudinal bidirectional creases on the overall shape of the membrane in their respective directions is discussed. The load amplitude, load time and load value are the same as those in Section 4 . The changes of $I_{x}$ and $I_{y}$ with the deployment force $T$ are shown in Fig. 15.

It can be seen from the Fig. 15, with the gradual increase of the longitudinal crease angle of the membrane, the initial value of the transverse displacement also increases, and its increasing amplitude is more obvious. When the longitudinal crease angles are $15^{\circ}, 30^{\circ}$, and $45^{\circ}$, the angles between adjacent transverse creases of the folded membrane are about $31.4^{\circ}, 64.1^{\circ}$, and $90.13^{\circ}$, respectively. It is the above 
angle change that causes the difference between the initial values of the transverse displacement. It is worth noting that the initial value of the longitudinal displacement of the membrane does not show a significant increase or decrease trend with the gradual increase of the longitudinal crease angle of the membrane, that is, it has little correlation with the longitudinal crease angle. However, the changing trend of the longitudinal displacement has been affected with the deployment of the folded membrane.

With the gradual increase of the longitudinal crease angle within the range of $15^{\circ}$ to $45^{\circ}$, the change rate of the longitudinal displacement under the same deployment force gradually decreases from the membrane deploying process. With the increase of the initial value of the transverse displacement, when $T \geq 0.2 \mathrm{~N} / \mathrm{mm}$, the synchronization between the transverse displacement and the longitudinal displacement of the membrane tends to be strengthened. For example, when $T=0.5 \mathrm{~N} / \mathrm{mm}$ and the membrane longitudinal crease angles are $15^{\circ}, 30^{\circ}$ and $45^{\circ}$ respectively, the difference between the transverse displacement and the longitudinal displacement is $17.7 \mathrm{~mm}, 10.83 \mathrm{~mm}$,

$2.82 \mathrm{~mm}$, respectively. It is worth noting that changing the longitudinal crease angle, the total length of the membrane crease is different. However, the limited amount of crease change does not affect the deployment force required for film deploying process too much.

\section{Deployment Experiment Of Miura Membrane}

In order to verify the reliability of the simulation results, a membrane with the longitudinal crease angle of $45^{\circ}$ and the number of parallelograms of $5 \times 5$ was taken as the research object, and the Miura folded membrane was deployed. The main quantity measured in the experiment are the transverse and longitudinal displacement of the folded membrane during the deploying process, and it is compared with the simulation results of the finite element method.

\subsection{Experimental design}

The experimental device is set up, as shown in Fig. 16. It mainly includes the overall diagram on the left side and the dismantling schematic diagram of each structure on the right side.

The main function of the precision optical vibration isolation platform is to isolate the influence of external interference in the experimental results in order to obtain relatively accurate experimental measurement values. There are regular $5 \mathrm{~mm}$ threaded holes on the platform, which can fix and build experimental components.

The hexagonal column is combined with the joint bolt and fixed on the precision optical vibration isolation platform. The two ends of connecting rod are respectively passed through the combination of two groups of hexagon columns and joint bolts to form the support frame. There are four groups of support frames and are arranged in a square.

The longitudinal crease of the membrane is $45^{\circ}$ and the number of parallelograms is $5 \times 5$. The black coating is uniformly arranged at the crease to make the overall configuration of the membrane more 
obvious during the deploying process. Small holes are punched at the four corners of the membrane, and the four small holes are respectively tied with cotton thread. The other end of the cotton thread is tied with a nylon thread with a diameter of $0.16 \mathrm{~mm}$. The nylon thread is placed on the support frame, and the weight is dropped to realize the application of the deployment force.

Among them, the cotton thread is used for transition in the membrane loading part to reduce the influence of the nylon thread that is too thin and tough. If the nylon thread is directly applied to the membrane, cracks will appear in the membrane, making the membrane unusable. The nylon thread with a diameter of $0.16 \mathrm{~mm}$ has a smooth surface, and the connecting rod contains a covering layer which causes the roughness to be small, so there is almost no friction in the contact between the above two. The folded membrane and weight are replaced at the same time. Since the size of the weight is variable, the application of different deployment forces can be realized. In addition, the folded membrane in this experimental method is only loaded with weights once and will not be used repeatedly, so the measured deployment results are more practical and instructive.

\subsection{Analysis of experimental influencing factors}

The main influencing factor of this experiment is whether the deployment force represented by the weight can be applied correctly. It is mainly divided into two aspects: First. as shown in Fig. 4, the deployment force parallel to the xoy plane is used in the membrane deployment simulation, which does not exist the component in the $z$ direction. Due to the light weight of the membrane, when the deployment force is applied as shown in Fig. 16, the membrane will be pulled up. And the nylon thread connected between the support frame and the cotton thread is almost parallel to the precision optical vibration isolation platform, so the error in this respect can be ignored. Second, the cotton thread is in contact with the connecting rod in the support frame assembly, and then static friction is generated. The last section said that the frictional resistance between the above two factors is very small, but it needs to be quantitatively discussed.

The experimental platform shown in Fig. 17 was built to discuss the influence of the friction between the nylon thread and the connecting rod. A digital display force gauge is used to measure the tension of the nylon thread. During the experiment, the digital display force gauge is always kept in a fixed state, and it is almost parallel to the surface of the precision optical vibration isolation platform.

In the case of a fixed weight, observe the numerical range of the digital display force gauge, and record it as shown in Table 3.

It can be seen from the table that the maximum error range of the mass measurement value of the weight caused by friction is between $-4.0 \%-+3.0 \%$, which means that the influence of friction on the deploying process of the membrane is small, and the experimental conditions meet the requirements.

Table 3 Analysis of experimental influencing factors. 


\begin{tabular}{lll} 
Weights $(\mathrm{g})$ & Range of measurement $(\mathrm{g})$ & Range of error \\
\hline 50 & $48-51$ & $-4.0 \%-+2.0 \%$ \\
\hline 100 & $97-103$ & $-3.0 \%-+3.0 \%$ \\
\hline 200 & $197-200$ & $-1.5 \%-0.0 \%$ \\
\hline 500 & $495-504$ & $-1.0 \%-+0.8 \%$ \\
\hline 700 & $696-701$ & $-0.6 \%-+0.2 \%$ \\
\hline 1000 & $989-1002$ & $-1.1 \%-+0.2 \%$
\end{tabular}

\subsection{Membrane deployment experiment}

The membrane of $210 \mathrm{~mm} \times 210 \mathrm{~mm}$ is folded in the Miura method. Folded height is one of the parameters to measure the degree of membrane folding. ${ }^{14}$ In order to ensure that the folded height of each crease is close to the thickness of the membrane, the folded membrane is compacted by a weight. And this state is kept for 24 hours. Then the plastic of membrane crease takes place. The folded membrane is shown in Fig. 18.

The four corners of the folded membrane are perforated respectively, and the weights are connected to make membrane deploy. By replacing the membrane and weight at the same time, the deployment configuration of the membrane under different deployment forces is obtained, part of which is shown in Fig. 19. The symmetrical state of the membrane is ensured by adjusting the length of the part of the nylon thread which is outside of the support frame. In order to compare the experimental result with the simulation result, it is noted that the linear load $F$ is calculated by deployment force and the length of the load boundary given in simulation.

The transverse and longitudinal displacements are extracted during the membrane deploying process and compared with the membrane deployment simulation to verify the reliability of the membrane deployment simulation results, as shown in Fig. 20.

It can be seen from the Fig. 20 that the maximum point of the difference in the transverse displacement of the membrane is about $F=0.5 \mathrm{~N} / \mathrm{mm}$, the difference is $5.48 \mathrm{~mm}$, and the simulation result has an error of $9.79 \%$ compared with the experiment. The maximum point of the difference in the longitudinal displacement of the membrane is about $F=0.2 \mathrm{~N} / \mathrm{mm}$, and the difference is $9.7 \mathrm{~mm}$. When the membrane is fully folded, the longitudinal displacement is only the membrane thickness, it is close to $0 \mathrm{~mm}$, so the

simulation result has an error of $5.71 \%$ compared with the experiment. In addition, the initial value of the longitudinal displacement is quite different, which is $4.84 \mathrm{~mm}$. In this case, the membrane may not be fully compacted in the experiment, so the degree of free rebound is too large. The errors of the transverse and longitudinal displacements are within a reasonable range, indicating that the membrane deployment simulation process is reliable. 


\section{Declarations}

This paper uses finite element simulation to study the folding and deploying process of the multi-element Miura membrane, which is mainly summarized as the following four points:

(1) The proxy model is used to replace the crease to realize the folding and deployment simulation of the membrane with the longitudinal crease angle of $15^{\circ}$ and the number of parallelogram elements of $5 \times 5$. A series of simulation results such as the stretch ratio, the maximum Mises stress and the wrinkle are discussed.

(2) The number of parallelogram elements of the same size membrane is set to $3 \times 3,5 \times 5$, and $7 \times 7$, and the membrane deployment simulation is carried out. The results show that with the increase of the number of parallelograms within the above range, the storage volume of the folded membrane gradually decreases. However, the crease length and the crease density gradually increase. Therefore, the membrane surface damage is more serious. In addition, the transverse and longitudinal bidirectional displacement movement is not synchronized during the deploying process of the membrane with different element numbers.

(3) The longitudinal crease angles of the membrane of the same size are set to $15^{\circ}, 30^{\circ}$, and $45^{\circ}$, and the membrane deployment simulation is carried out. The results show that with the increase of the longitudinal crease angle within the above range, the storage volume of the folded membrane and the crease length gradually increases. However, the crease density does not increase, and there is an upper limit to the degree of membrane surfaces damage. In addition, the movement synchronization of the transverse and longitudinal displacements during the membrane deploying process gradually increases with the gradual increase of the longitudinal crease angle.

(4) The deployment experiment is carried out on the folded membrane. The experimental platform is designed. And the experimental influence factors are analyzed. Finally, it proved that the deploying process simulation of the Miura membrane is reliable.

\section{Declarations}

\section{Acknowledgements}

Not applicable

\section{Authors' contributions}

JZ was in charge of the whole trial; YS wrote the manuscript; JZ and YS assisted with simulation and analyses. All authors read and approved the final manuscript.

\section{Authors' information}


Jing Zhang, born in 1985, is currently an associate professor at Taiyuan University of Technology, China. She received her PhD degree from Harbin Institute of Technology, China, in 2014. Her research interests include deployable structure and mechanism.

Youqing Shen, born in 1995, is currently a master candidate at Taiyuan University of Technology, China.

Hongwei Guo, born in 1980, is currently a professor at Harbin Institute of Technology. He received his PhD degree from Harbin Institute of Technology, China, in 2009.

Rongqiang Liu, born in 1965, is currently a professor at Harbin Institute of Technology. He received his PhD degree from Harbin Institute of Technology, China, in 1995.

Ziming Kou, born in 1964, is currently a professor at Taiyuan University of Technology, China. He received his PhD degree from China University of Mining and Technology, China, in 1995.

\section{Funding}

This work was supported by National Natural Science Foundation of China (Project Nos. 51835002), Natural Science Foundation of Shanxi Province (Project No. 201901D211010) and Technology Innovation Foundation of Shanxi University (Project No. 2019L0177).

\section{Competing interests}

The authors declare no competing financial interests.

\section{Author Details}

${ }^{1}$ College of Mechanical and Vehicle Engineering, Taiyuan University of Technology, Taiyuan, 030024, China. ${ }^{2}$ Shanxi Province Engineering Technology Research Center for Mine Fluid Control, Taiyuan, 030024, China. ${ }^{3}$ National-local Joint Engineering Laboratory of Mining Fluid Control, Taiyuan, 030024, China. ${ }^{4}$ School of Mechatronics Engineering, Harbin Institute of Technology, Harbin, 150001, China.

\section{References}

1. Fang H, Knarr K, Quijano U. In-space development reflectarray antenna: Current and future. The 49th AIAA/ASME/ASCE Structures, Structural dynamics and Material Conference, 2008.

2. Mori O, Sawada H, Funase R, et al. First solar power sail demonstration by IKAROS. Transactions of the Japanese Society for Artificial Intelligence 2011;8:425-431.

3. Hossain, Woo k, Jenkins $C$. Nonlinear material response of systematically creased membranes. Proc. of 47th AIAA/ASME/ASCE/AHS/ASC Structures, Structural Dynamics, and Materials Conference; 2006; Rhode Island, USA.

4. Okuizumi N, Yamamoto T. Centrifugal deployment of membrane with spiral folding: experiment and simulation. Journal of Space Engineering 2009;2(1):41-50. 
5. Murphey TW. A nonlinear elastic constitutive model for wrinkled thin films. University of Colorado 2000:1-133.

6. Mierunalan S, Mallikarachchi HMYC. Prediction of creased geometry of thin folded membranes using finite element analysis. Proc. of Moratuwa Engineering Research Conference (MERCon); 2017; Moratuwa, Sri Lanka.

7. Satou Y, Furuya H. Effects of elasto-plastic behavior of crease on deployed shape of space membrane. Proc. of AIAA Spacecraft Structures Conference, 2018; Florida, USA.

8. Xia Z, Wang C, Tan H F. Quasi-static unfolding mechanics of a creased membrane based on a finite deformation crease-beam model. Int J Solids Struct 2020;207(15):104-112.

9. Turner N, Goodwine B, Sen M. A review of origami applications in mechanical engineering. Proc Inst Mech Eng Part C-J Eng Mech Eng Sci 2015;230(14):2345-2362.

10. Papa A, Pellegrino S. Systematically Creased Thin-Film Membrane Structures. J Spacecr Rockets 2008;45(1):10-18.

11. Xu Y, Guan FL, Fold methods and deployment analysis of deployable membrane structure. Engineering Mechanics 2008;5:176-181.

12. Arya M, Pellegrino S. Deployment mechanics of highly compacted thin membrane structures. Spacecraft Structures Conference, 2014.

13. Cai JG, Ren Z, Ding YF, et al. Deployment simulation of foldable origami membrane structures. Aerosp Sci Technol 2017;67:343-353.

14. Shen YQ, Zhang J, Guo HW, et al. Deployment simulation of membrane structures based on elasticplastic behavior parameterization of the crease. J Mech Sci Technol 2021;35(5).

15. Satou Y, Furuya H. Mechanical properties of z-fold membrane under elasto-plastic deformation. Journal of Space Engineering 2011;4(4):14-26.

16. Tong ZY, Li M, Cui CB, et al. Design and Analysis of the Configuration of Deployable Membrane Sunsheild. Chinese Space Science and Technology 1-7. Available from: http://kns.cnki.net/kcms/detail/11.1859.V.20201112.1738.002.html.

17. Wang CG, Xie J, Tan HF. Wrinkling characteristics analysis of inflated membrane arch. Journal of Harbin Institute of Technology 2013;45(03):66-69.

18. Wong YW, Pellegrino S. Wrinkled membranes part $₫$ : Numerical Simulations. J Mech Mater Struct 2006;1(1):61-93.

\section{Figures}




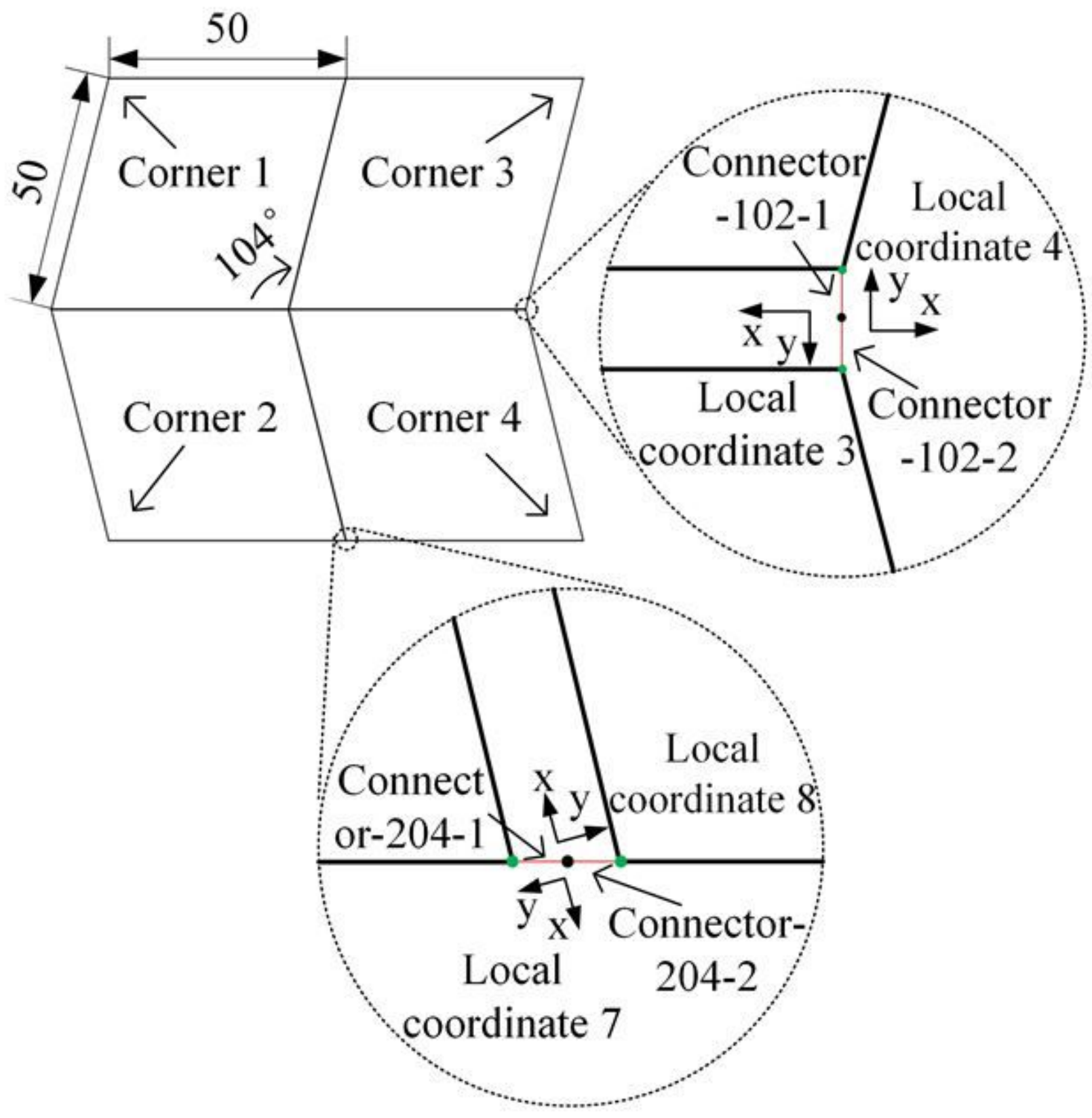

Figure 1

The method of crease substitution 14 


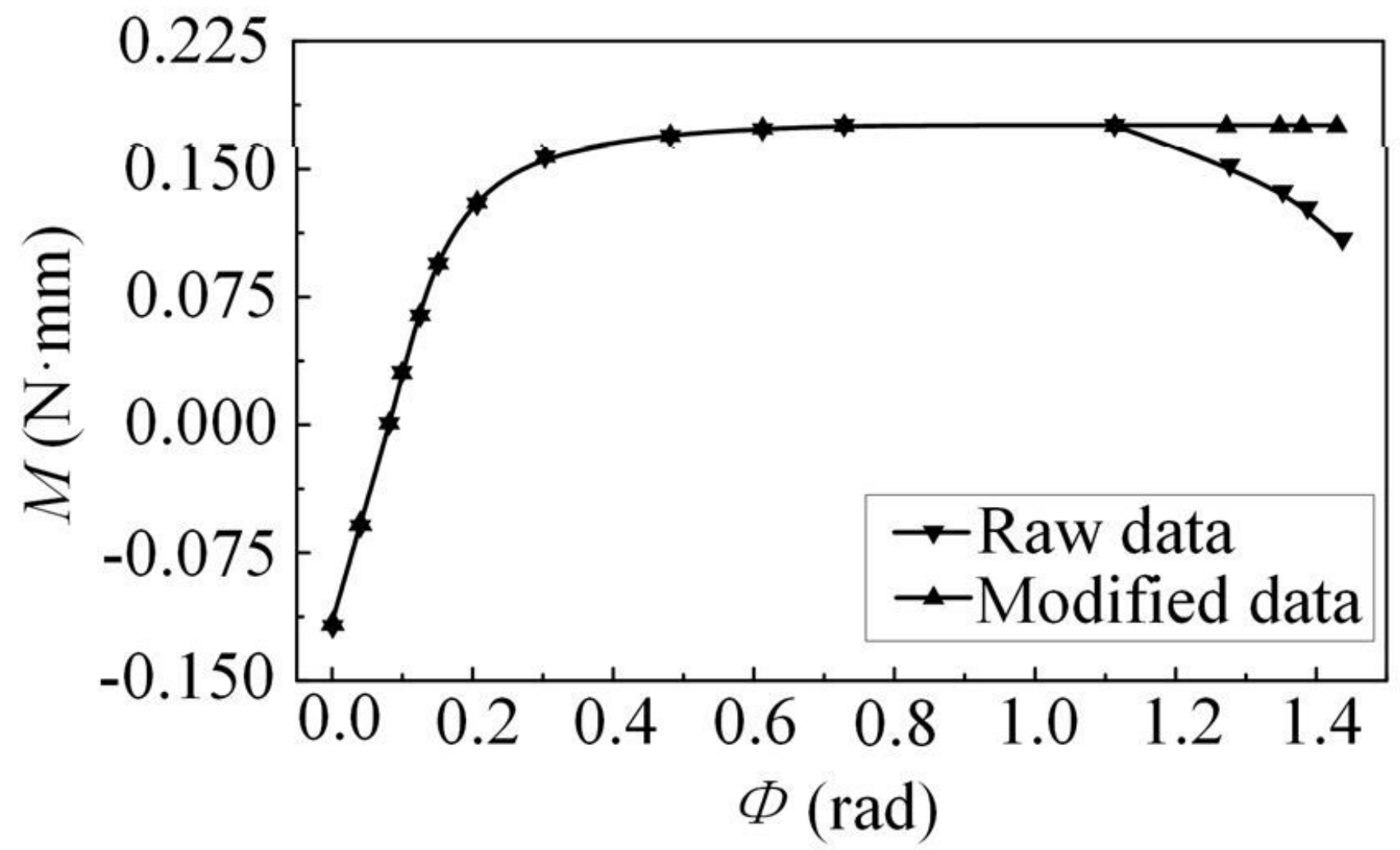

Figure 2

Elastic-plastic behavior of the crease 14

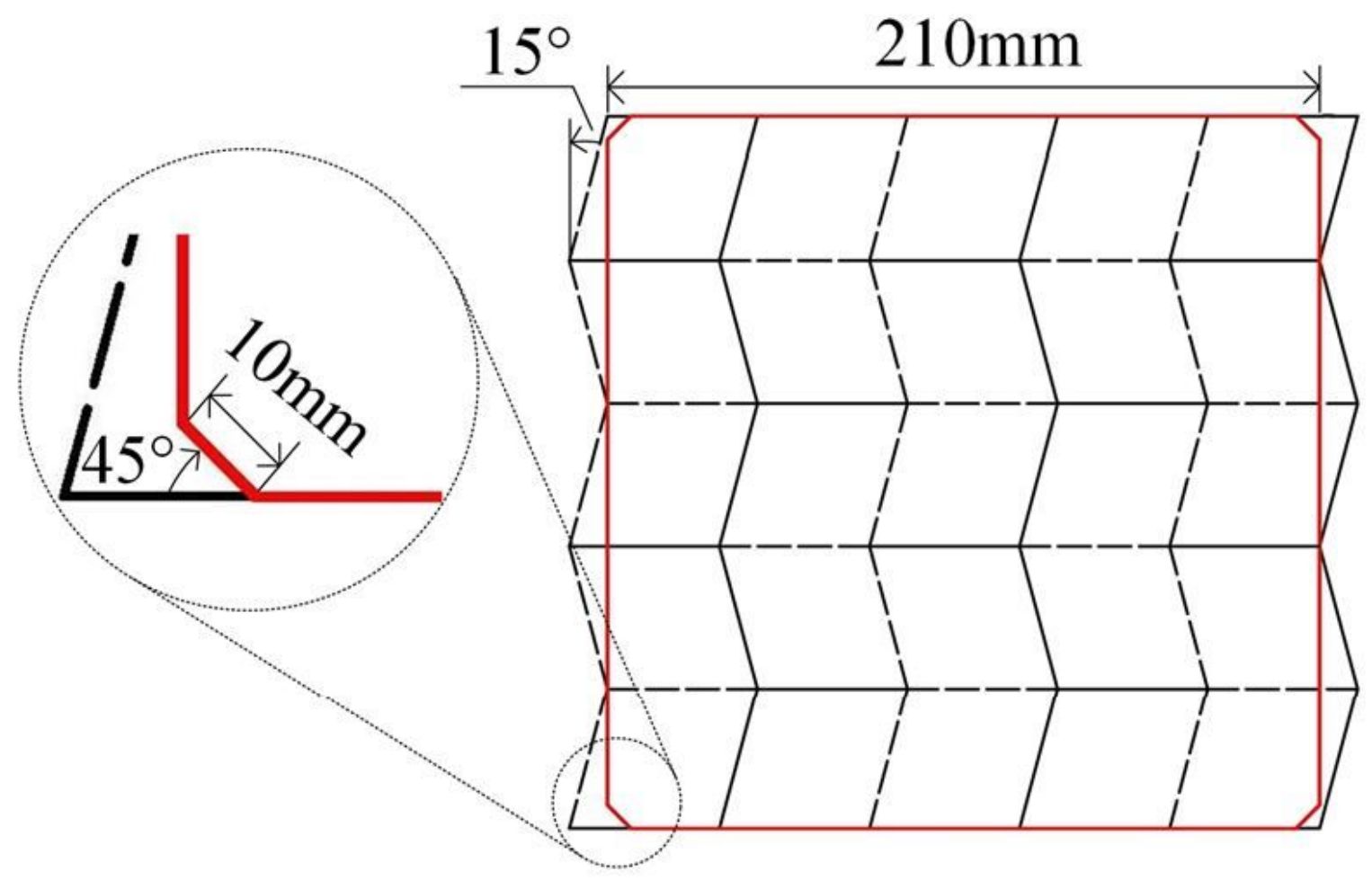


Figure 3

Modeling of Miura membrane

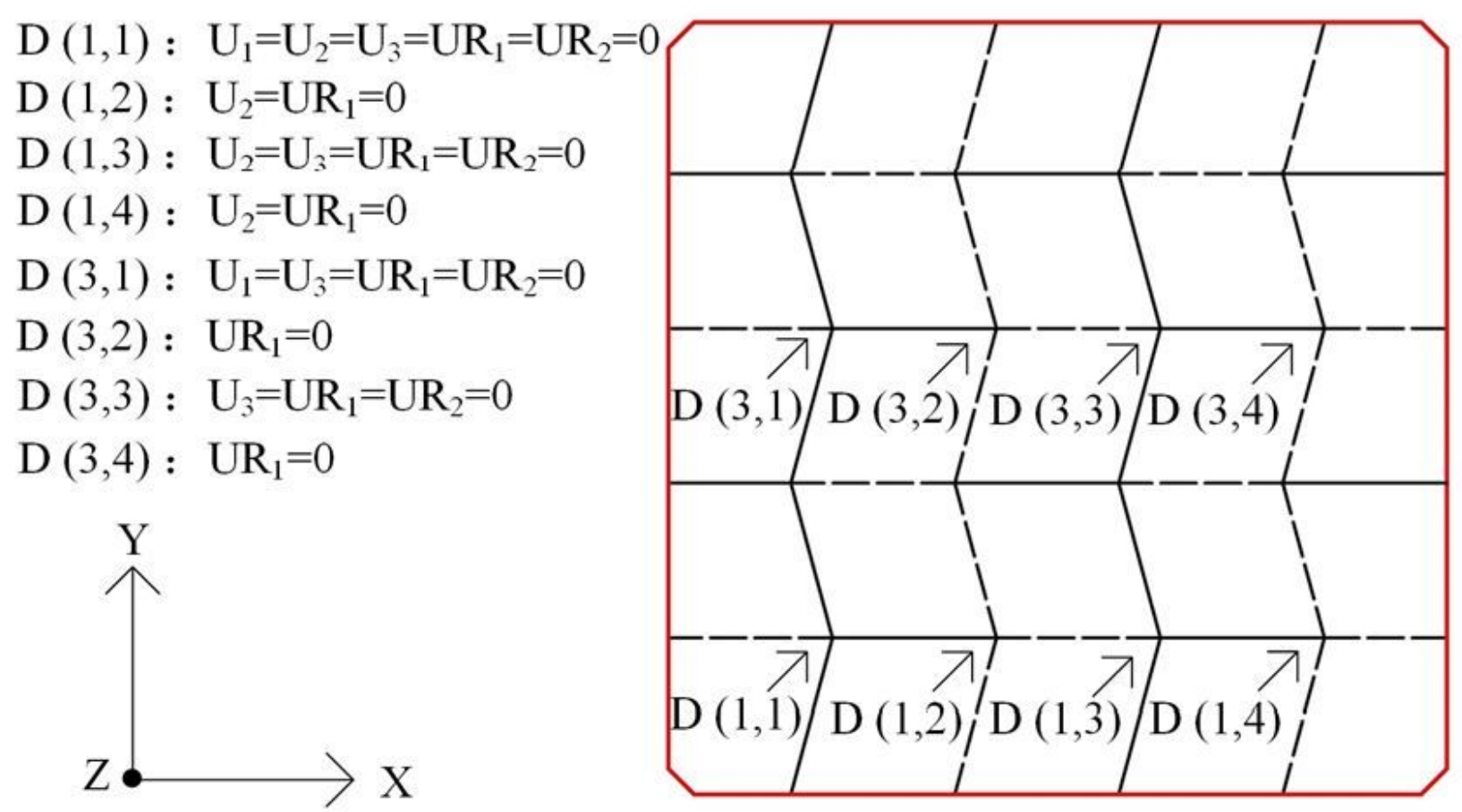

Figure 4

Constraints of the membrane 


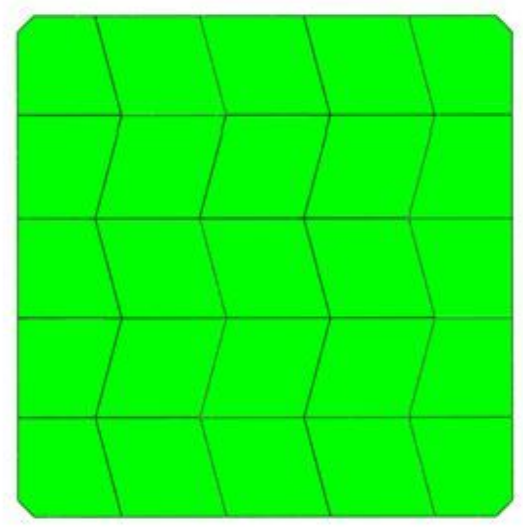

(a) $0 \mathrm{~s}$

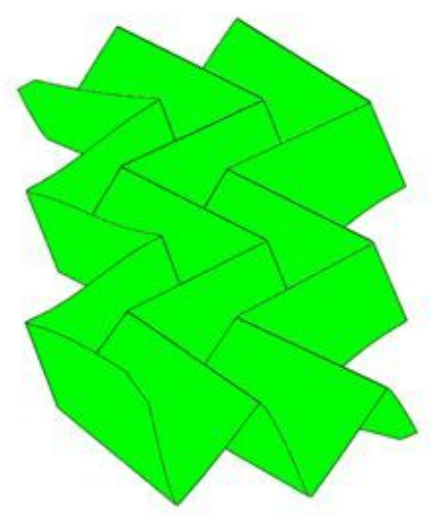

(c) $0.07 \mathrm{~s}$

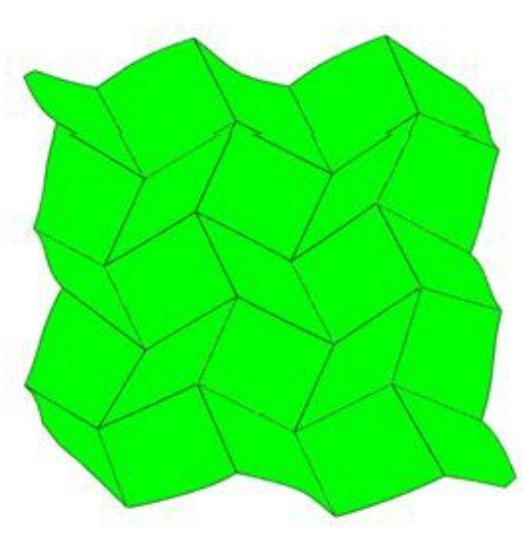

(b) $0.035 \mathrm{~s}$

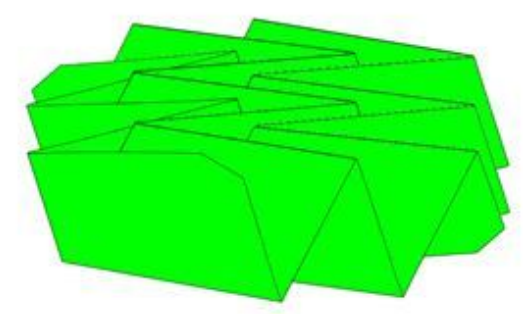

(d) $0.1 \mathrm{~s}$
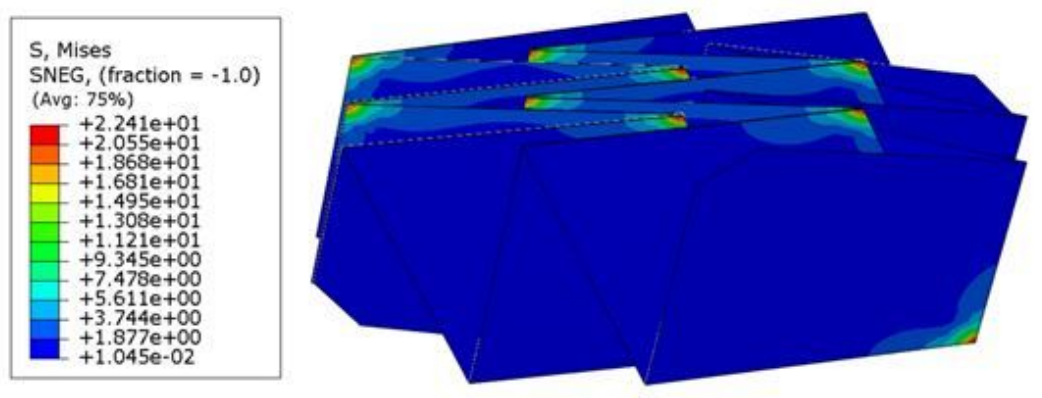

(e) $1 \mathrm{~s}$

Figure 5

Membrane folding process 

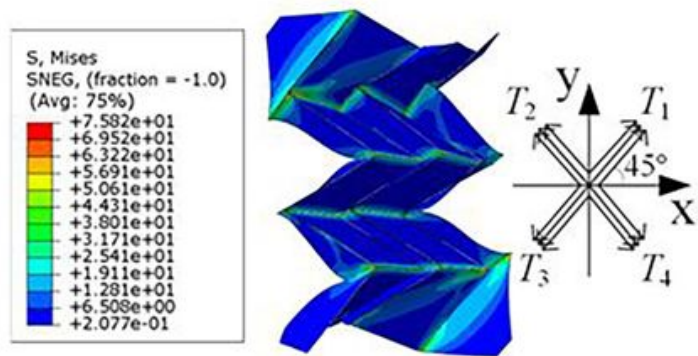

(a) $T=0.05 \mathrm{~N} / \mathrm{mm}$
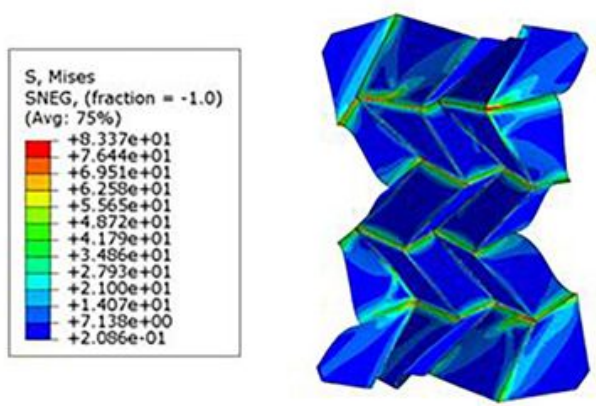

(b) $T=0.1 \mathrm{~N} / \mathrm{mm}$
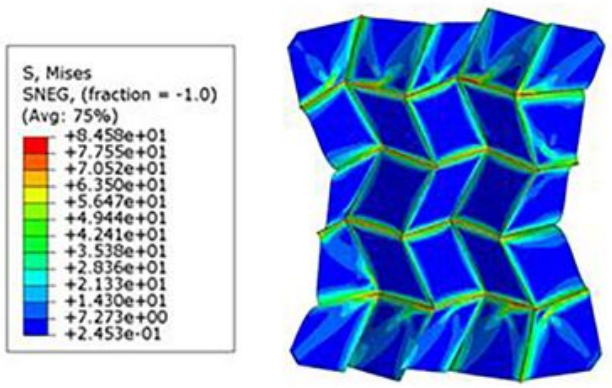

(c) $T=0.15 \mathrm{~N} / \mathrm{mm}$
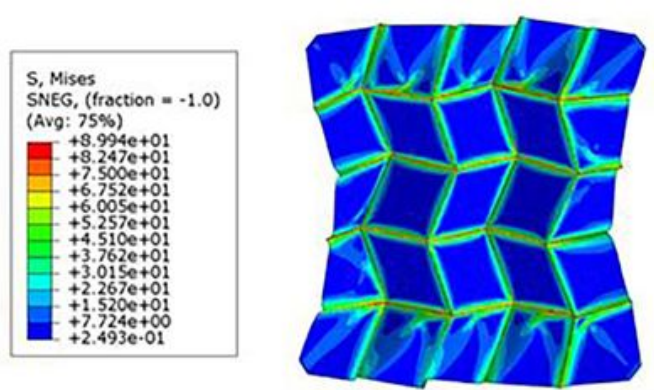

(d) $T=0.2 \mathrm{~N} / \mathrm{mm}$
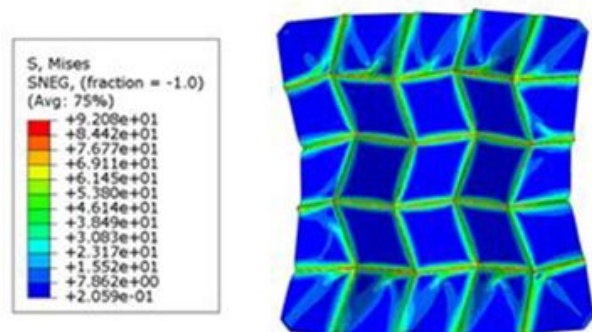

(e) $T=0.25 \mathrm{~N} / \mathrm{mm}$
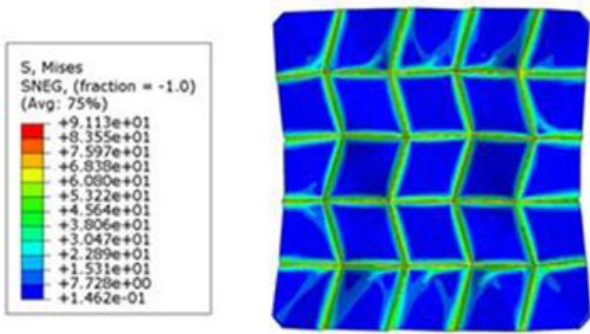

(f) $T=0.5 \mathrm{~N} / \mathrm{mm}$
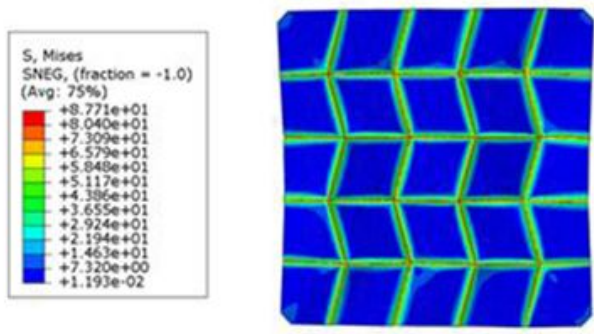

(g) $T=1 \mathrm{~N} / \mathrm{mm}$
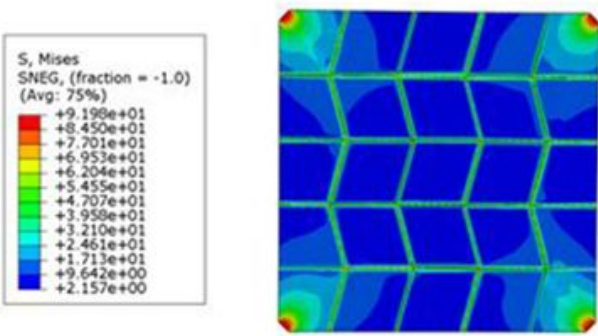

(h) $T=10 \mathrm{~N} / \mathrm{mm}$

Figure 6

Membrane deploying process 


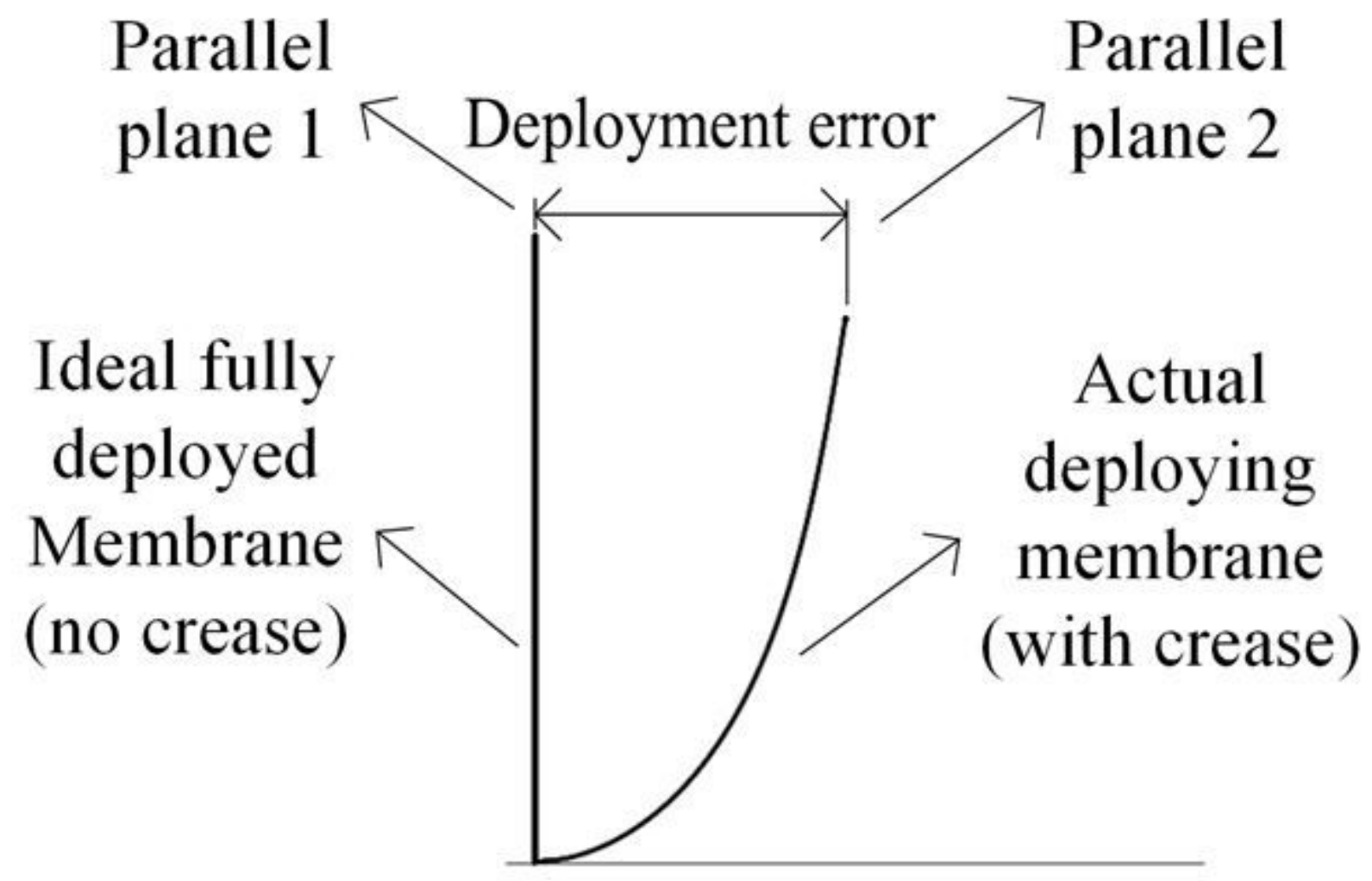

Figure 7

Definition of deployment error 14 


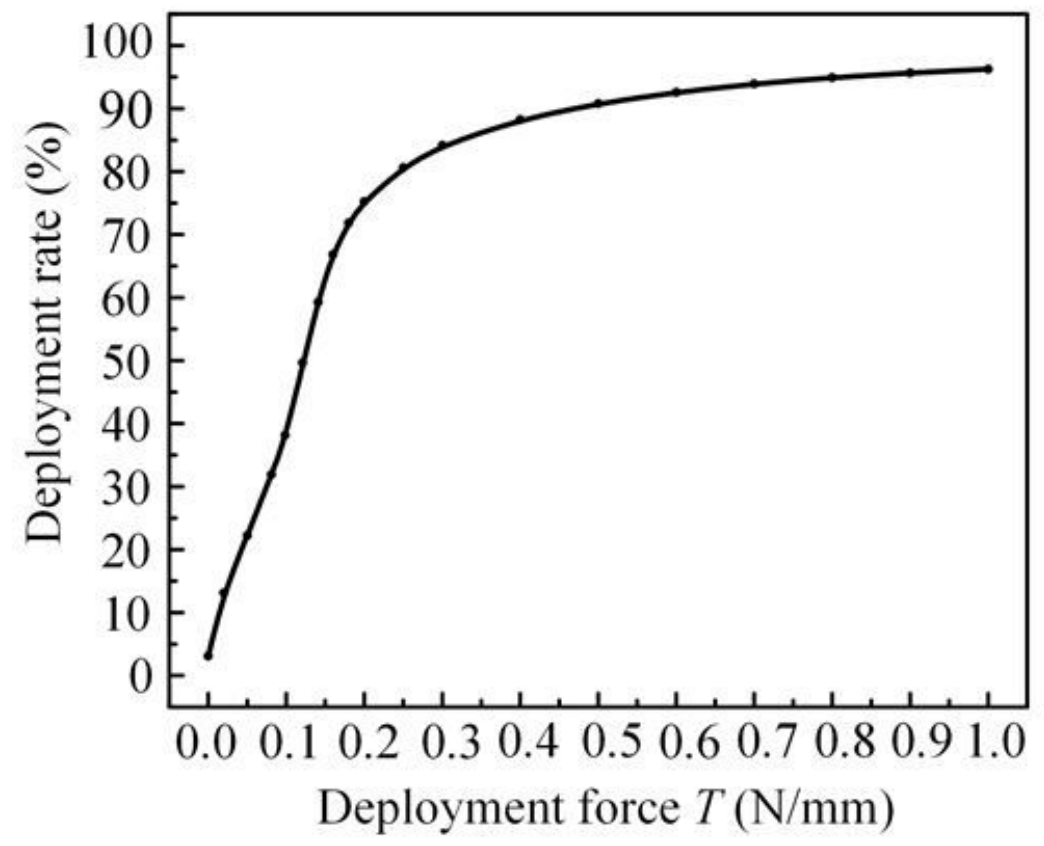

(a) Variation curve of deployment rate

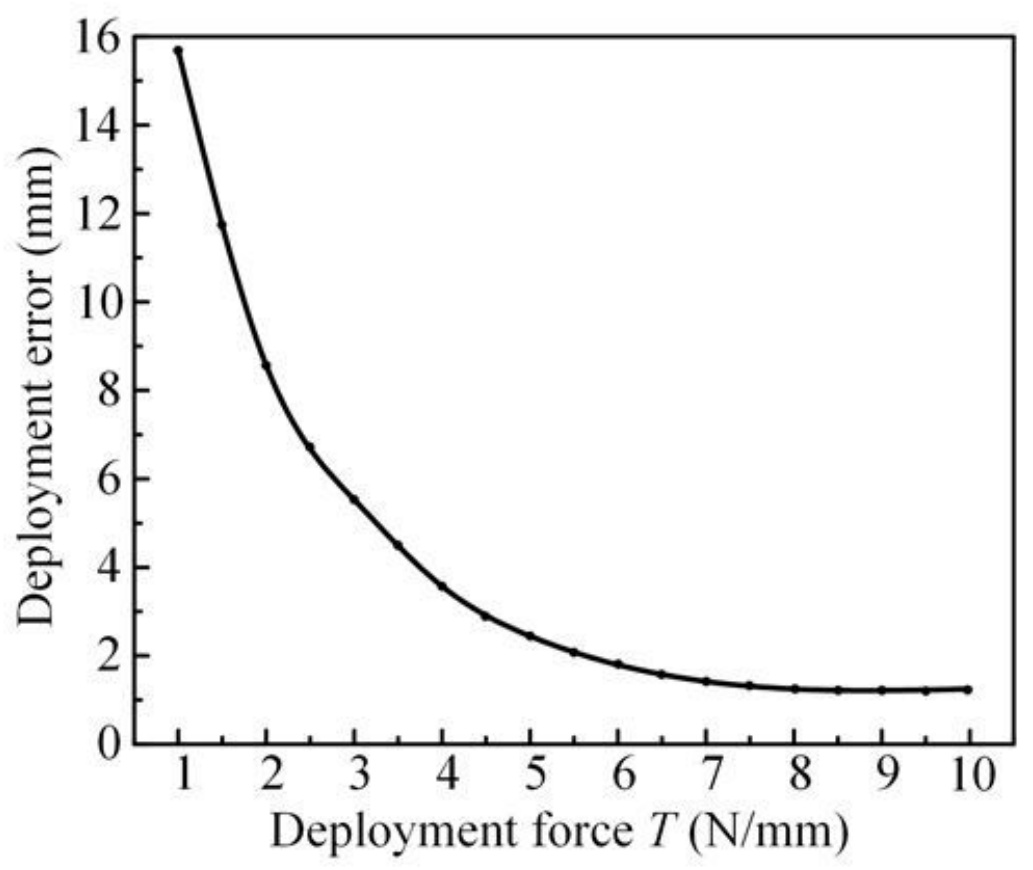

Figure 8

The deployment rate and deployment error 


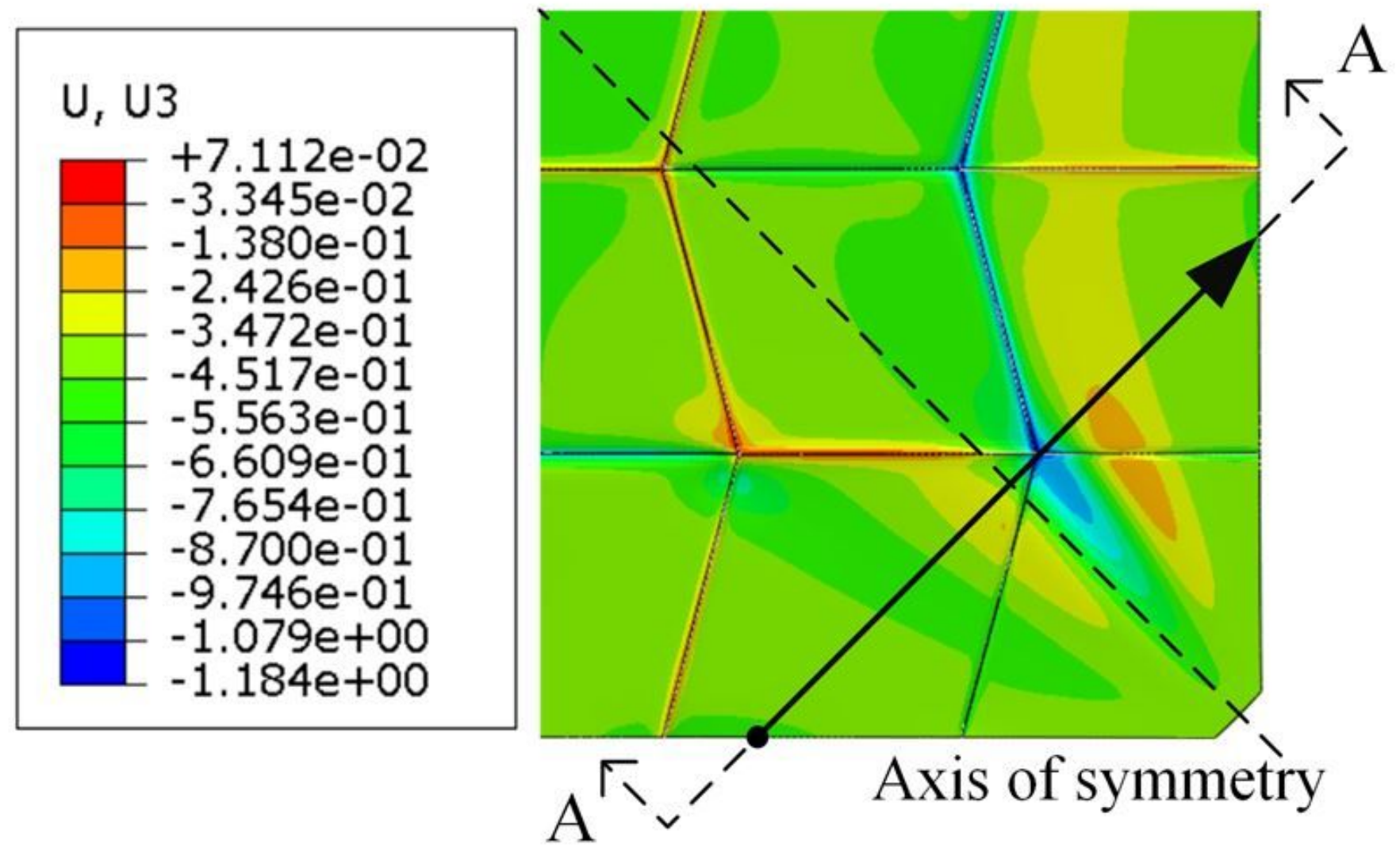

Figure 9

Displacement diagram of the membrane in the Z-axis direction 


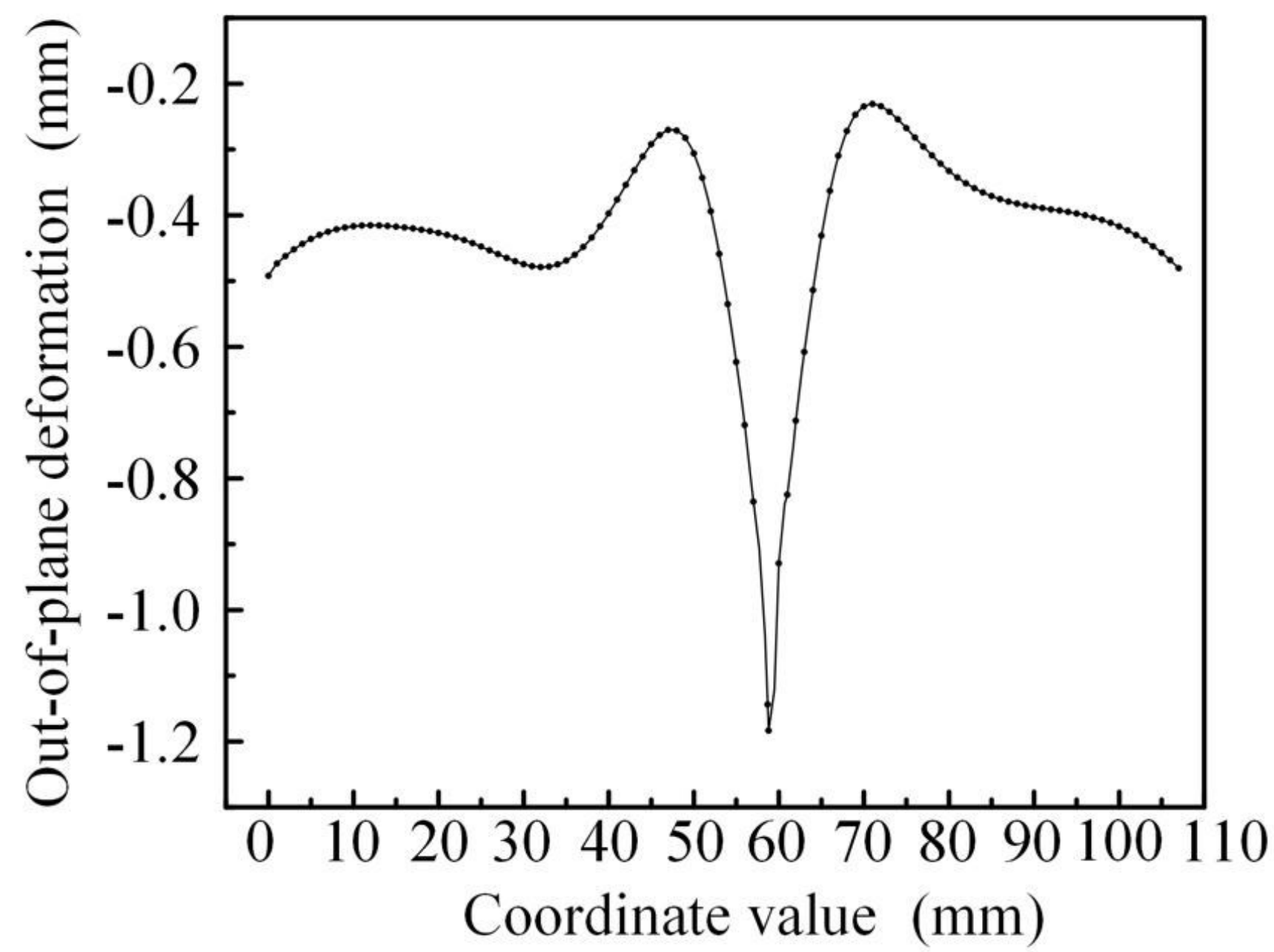

Figure 10

Out-of-plane deformation of the membrane 


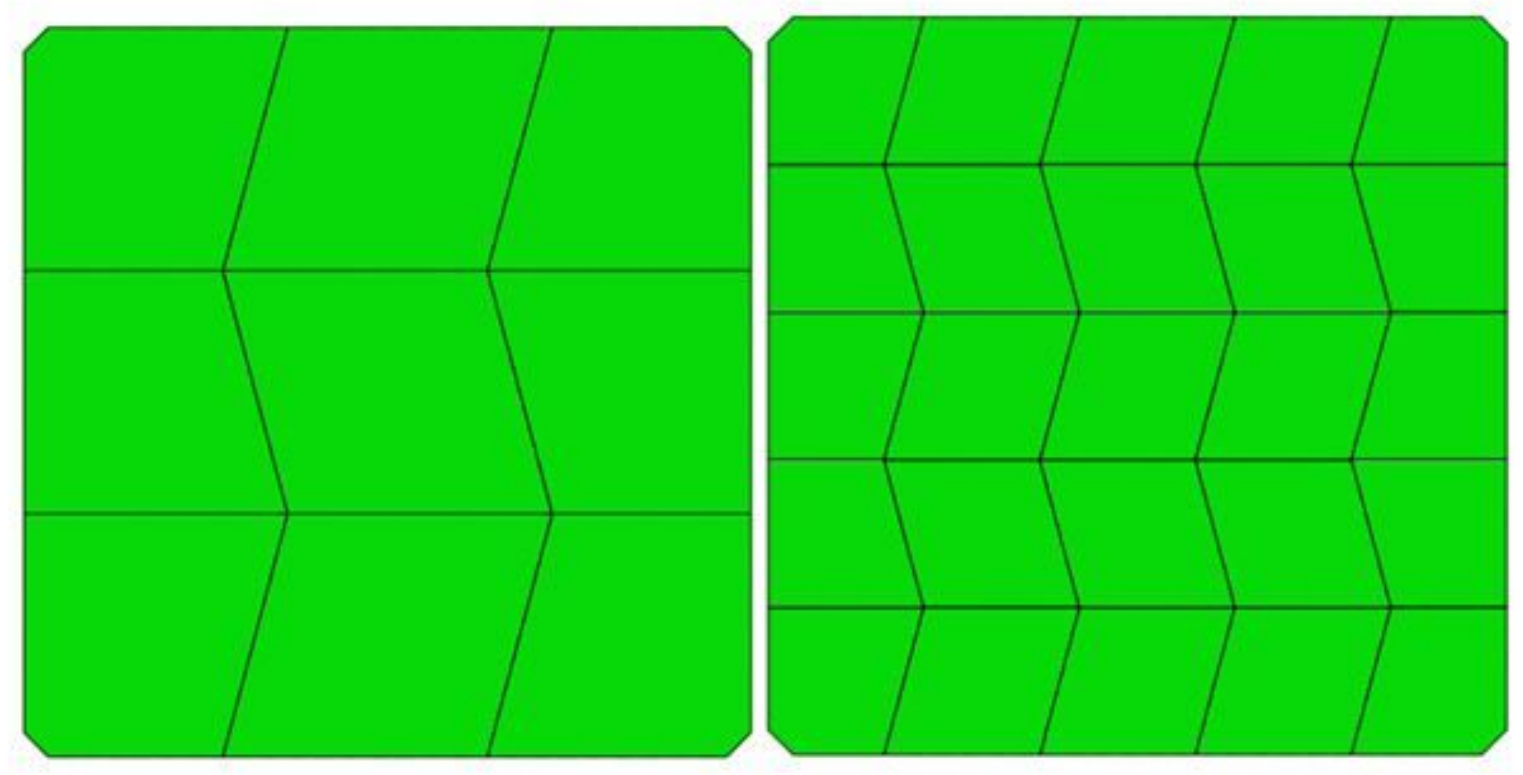

(a) $3 \times 3$

(b) $5 \times 5$

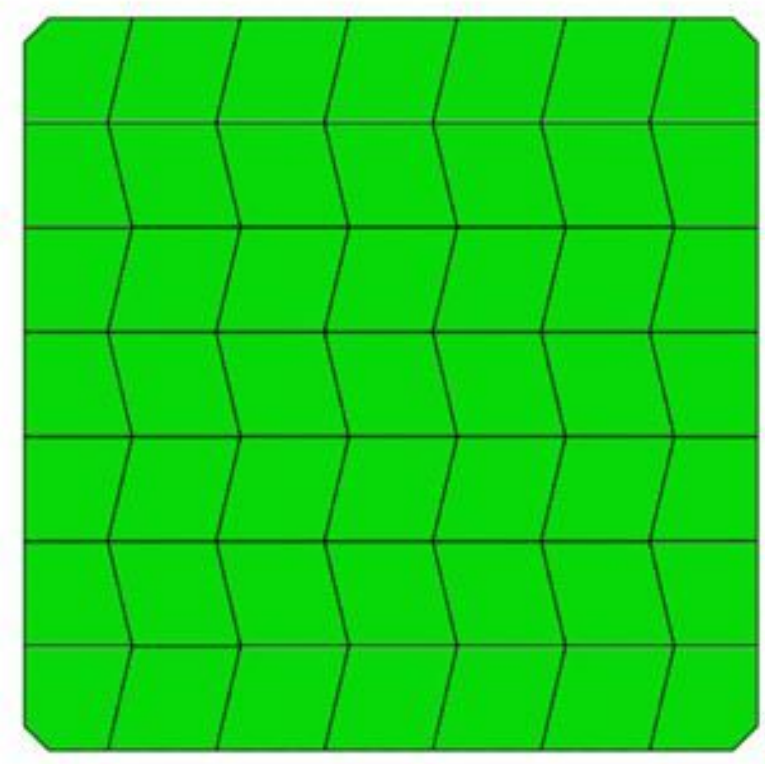

(c) $7 \times 7$

Figure 11

Membranes of different numbers of elements 


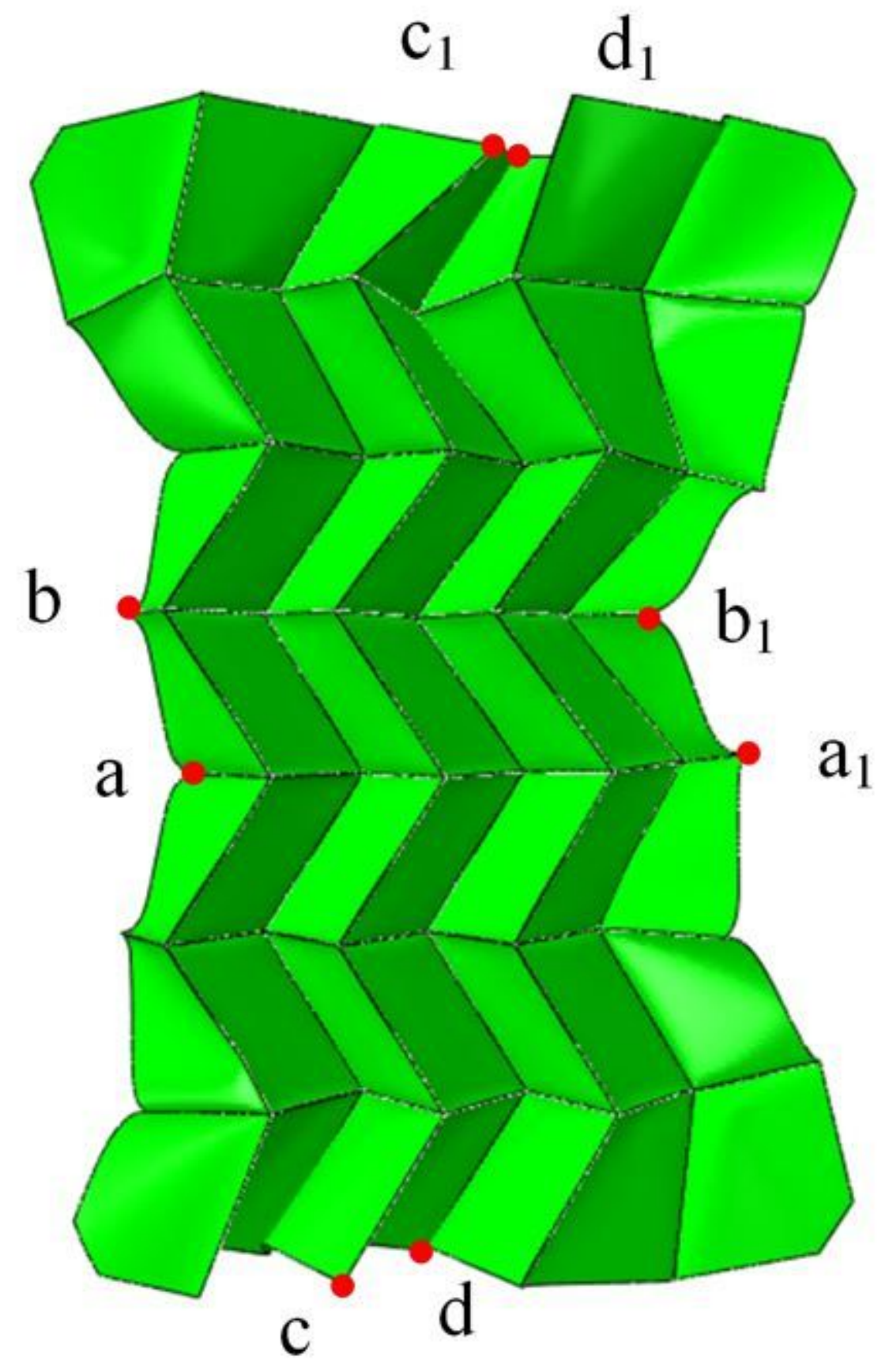

Figure 12

The deploying process of $7 \times 7$ membrane 


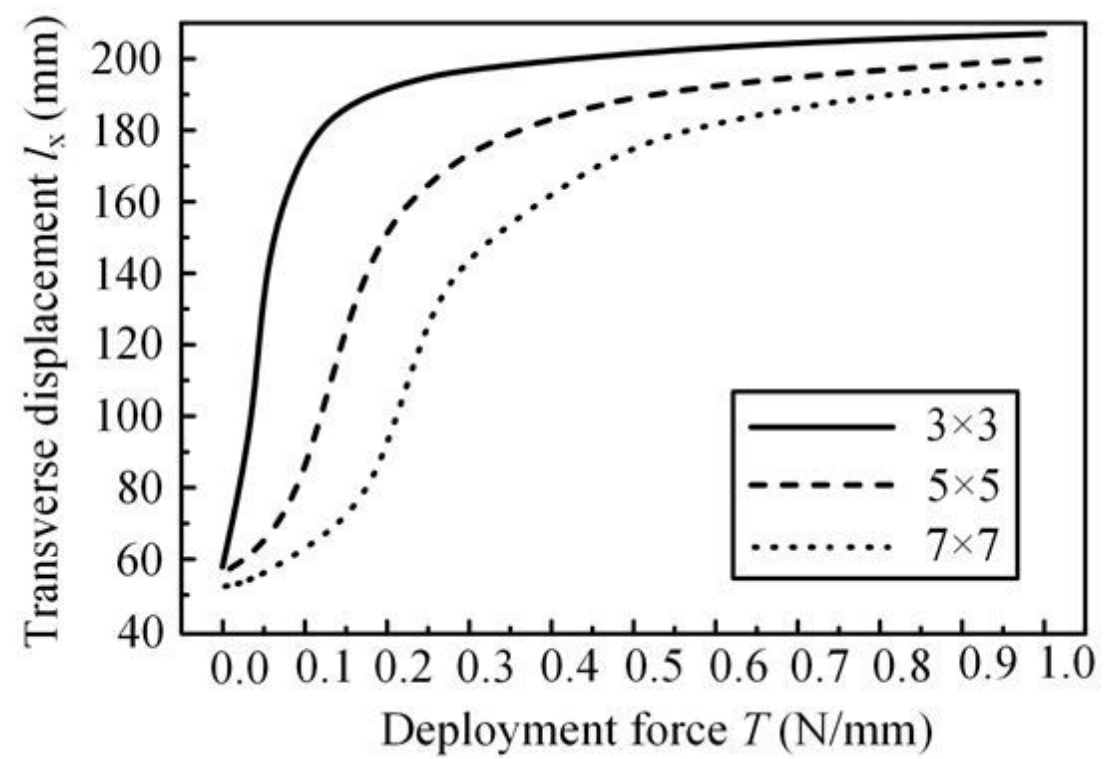

(a) The transverse displacement

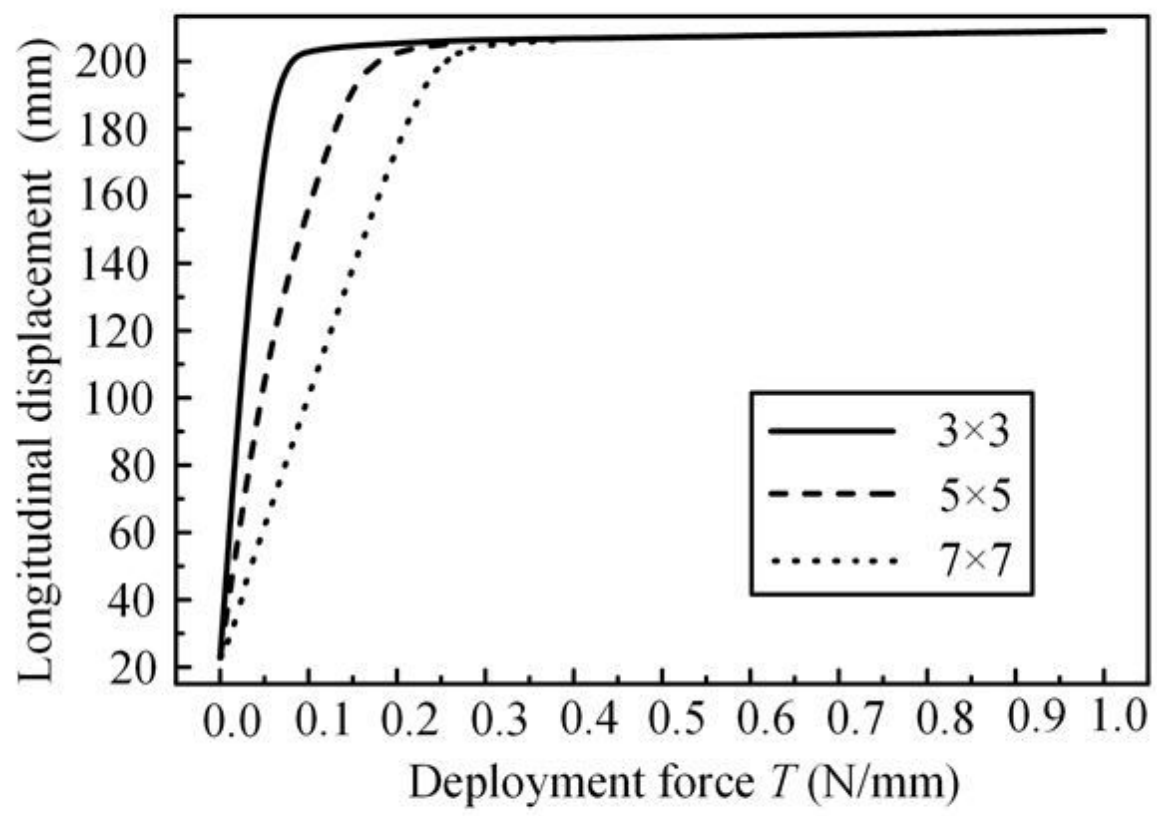

(b) The longitudinal displacement

Figure 13

Variation curves of transverse and longitudinal displacements 


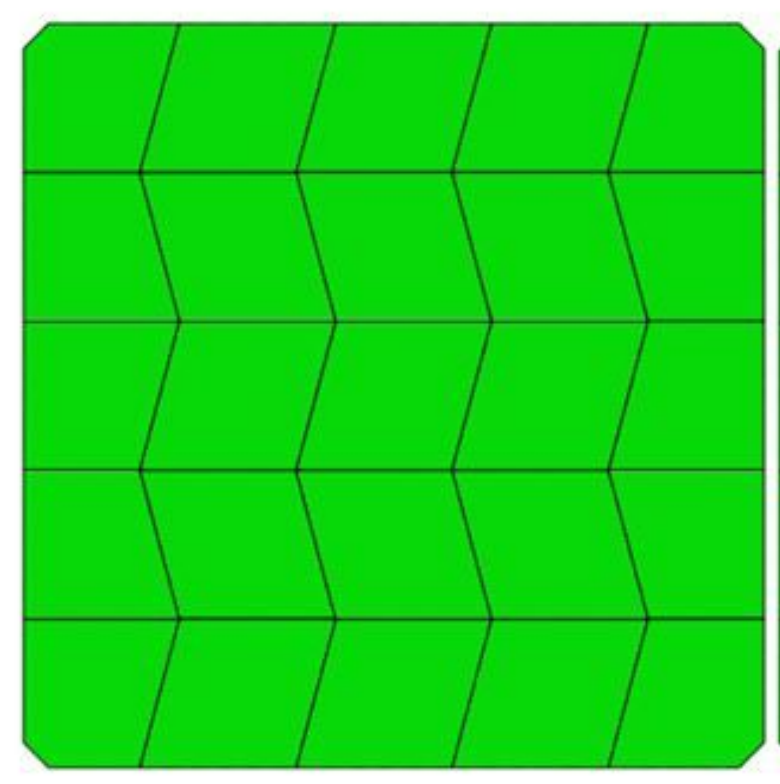

(a) $15^{\circ}$

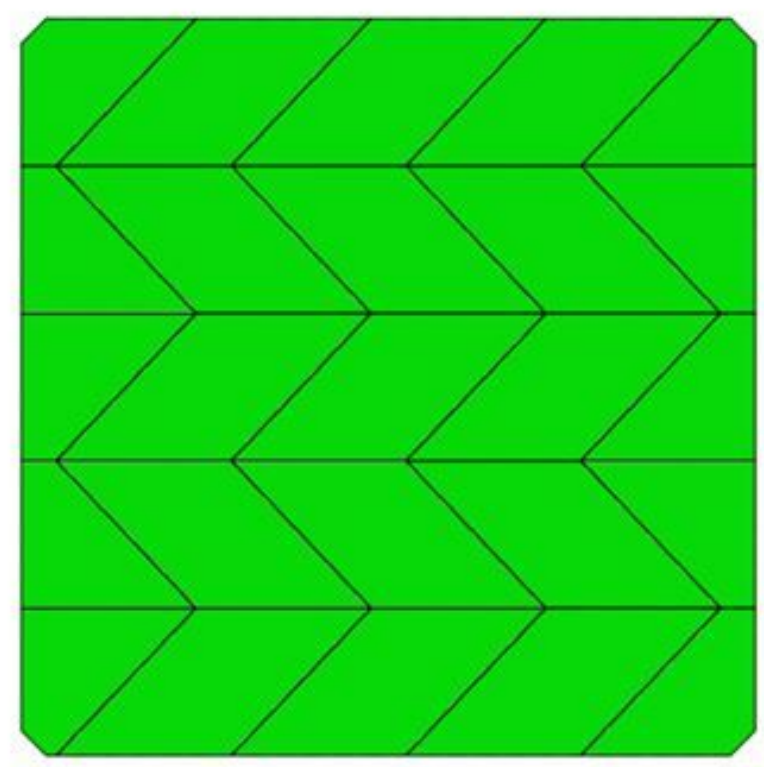

(c) $45^{\circ}$

Figure 14

Membranes with different longitudinal crease angles 


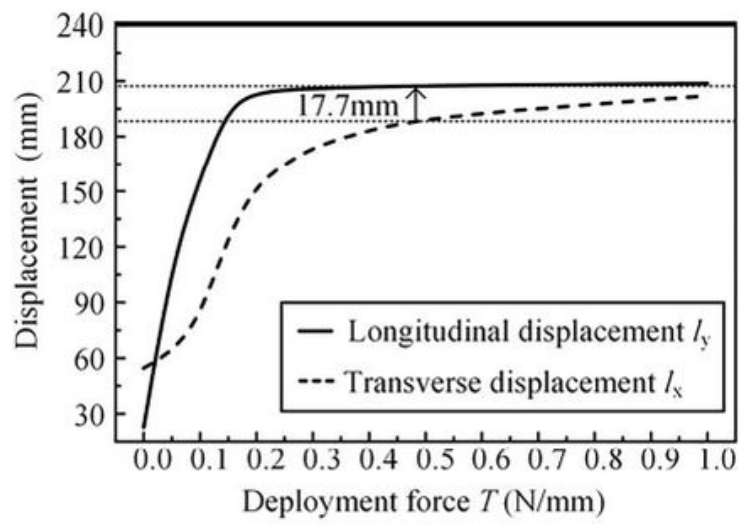

(a) $15^{\circ}$

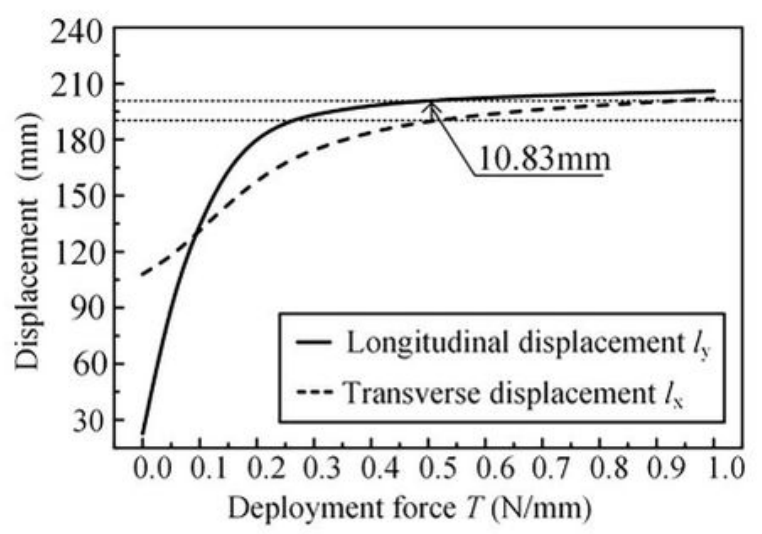

(b) $30^{\circ}$

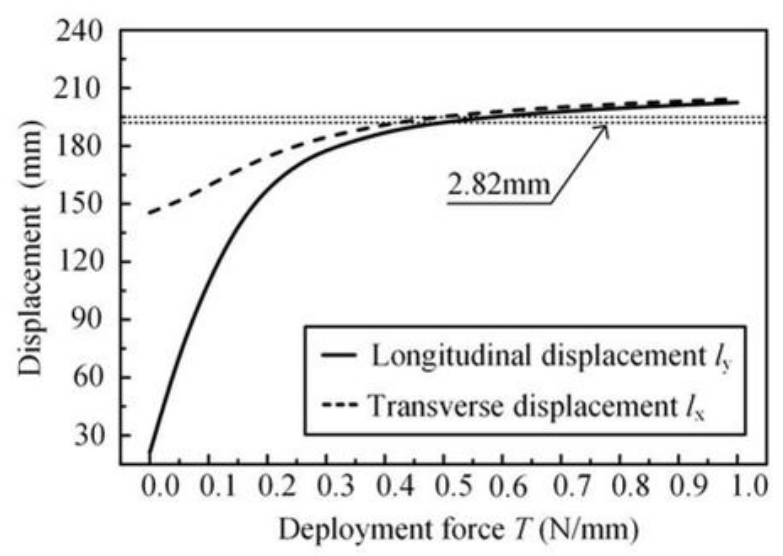

(c) $45^{\circ}$

\section{Figure 15}

Variation curves with different longitudinal crease angle 

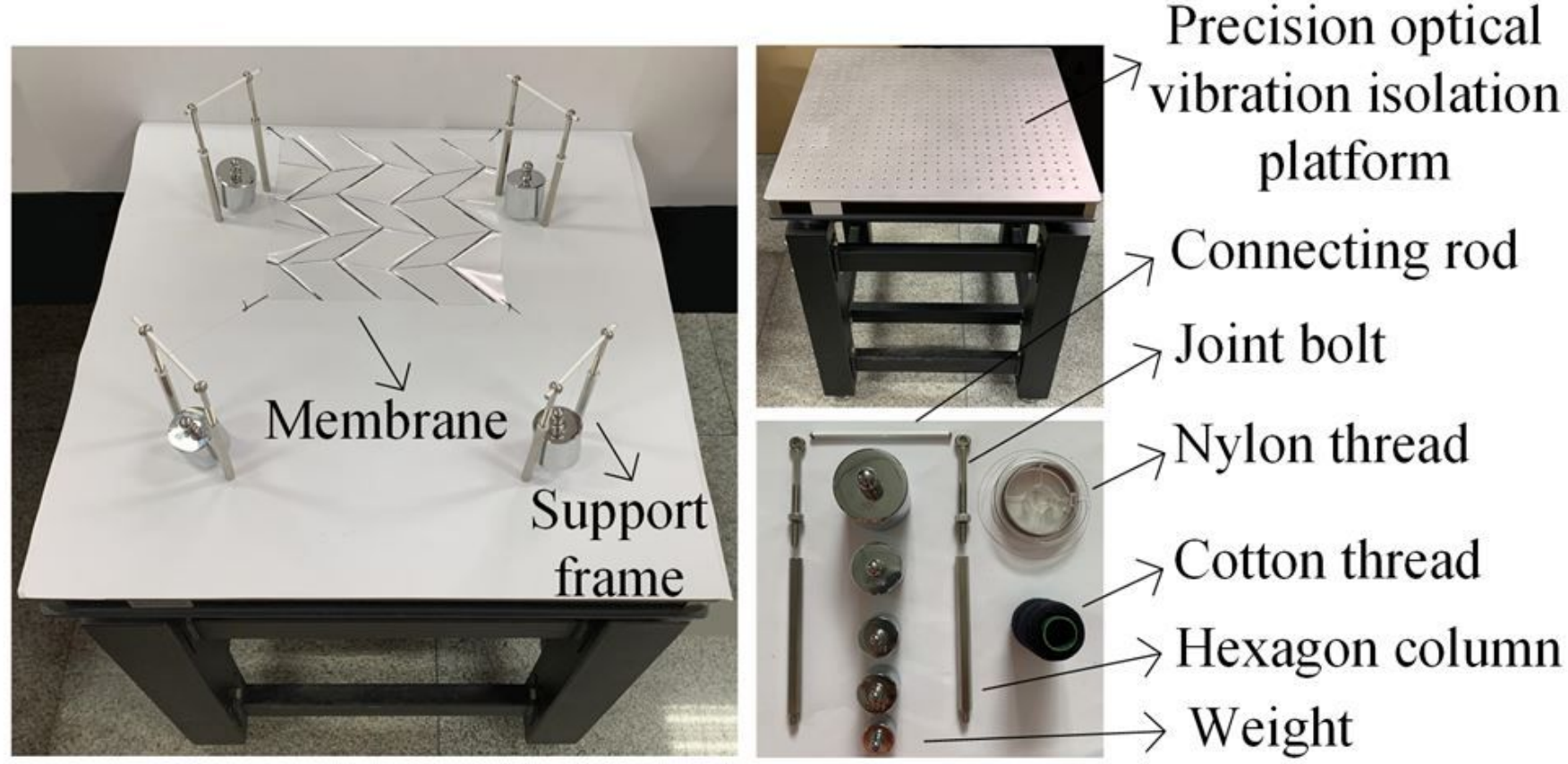

Figure 16

Experimental platform design 

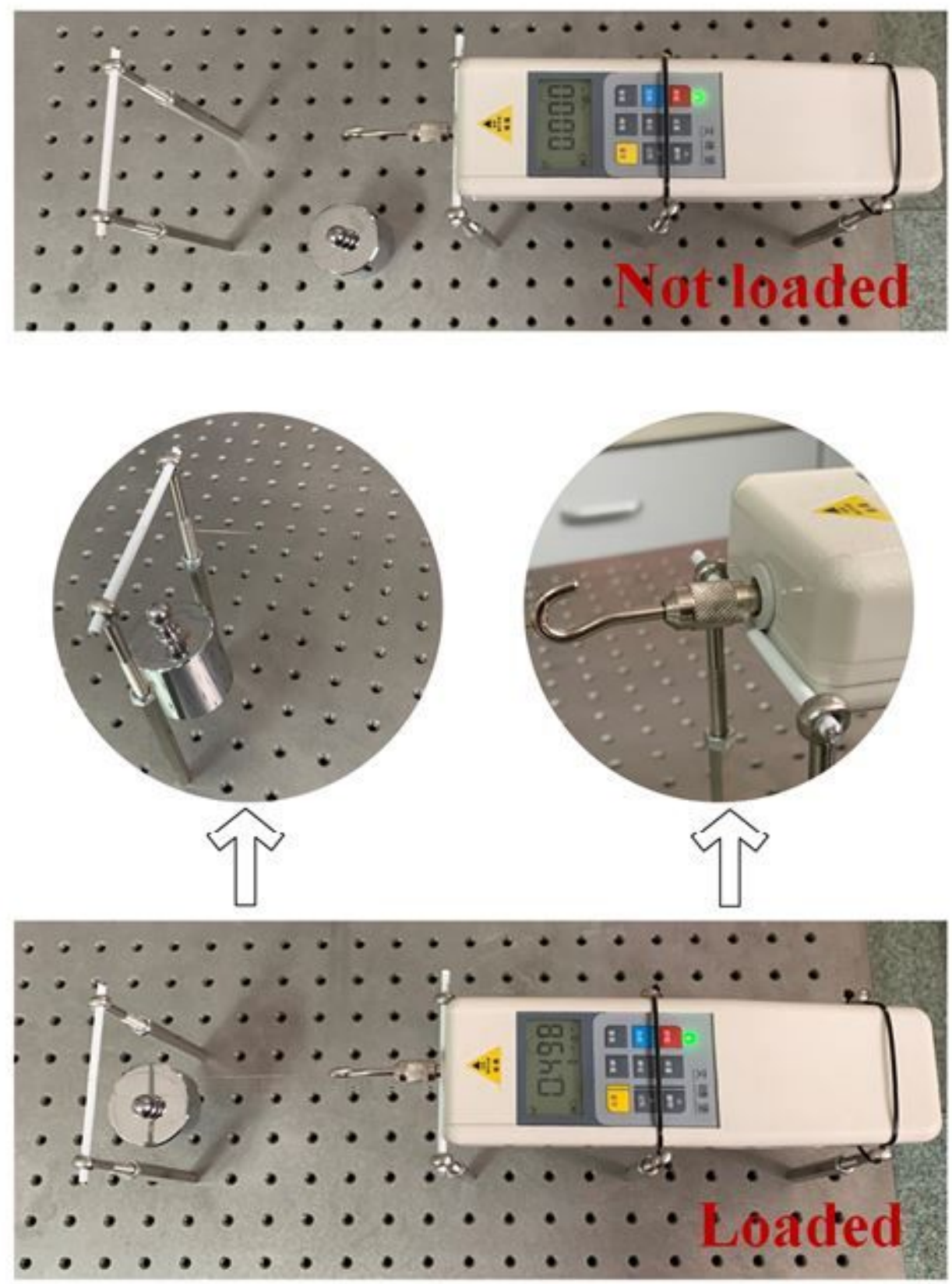

Figure 17

The experiment of error analysis 


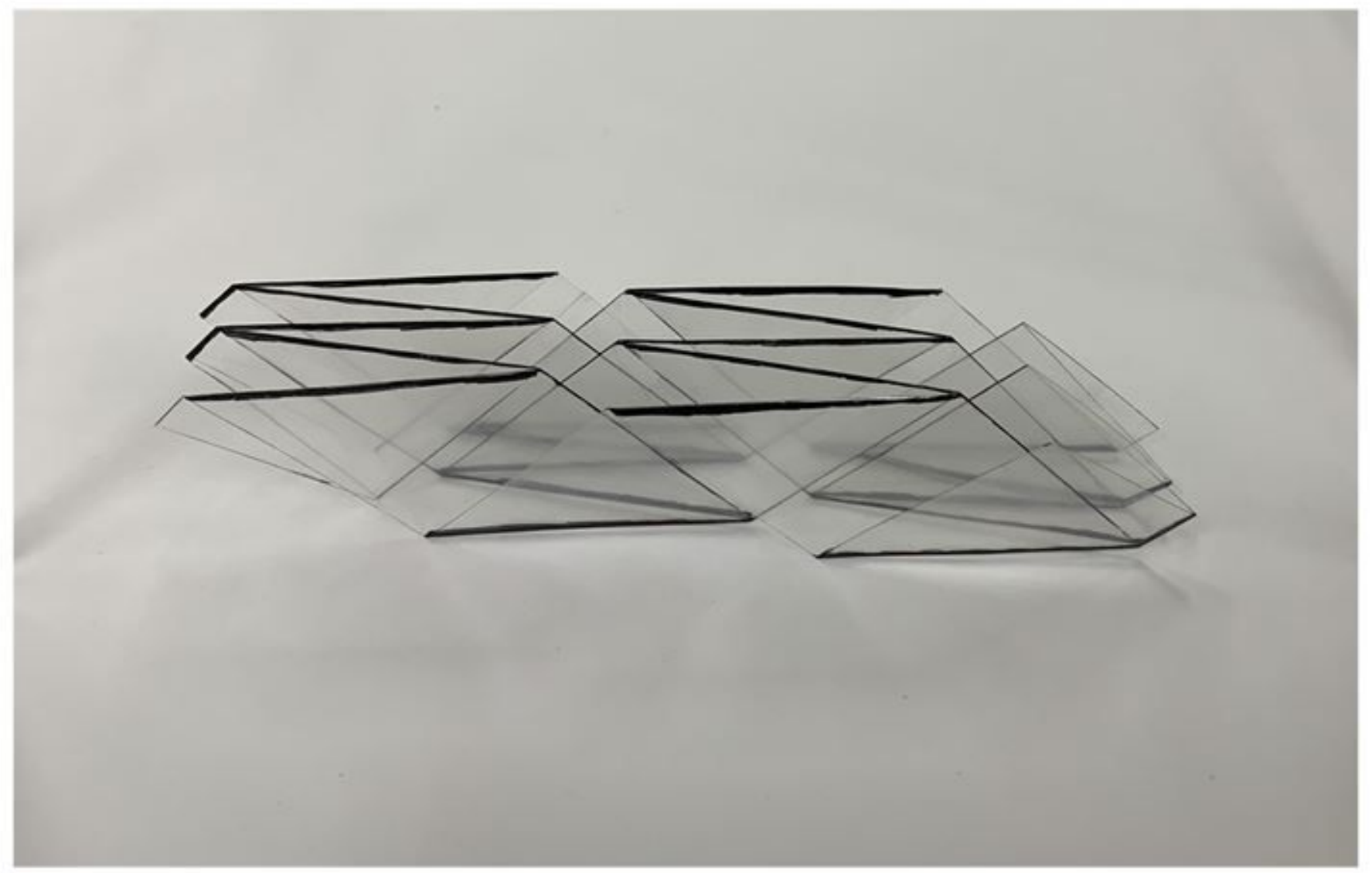

Figure 18

Folded configuration membrane 


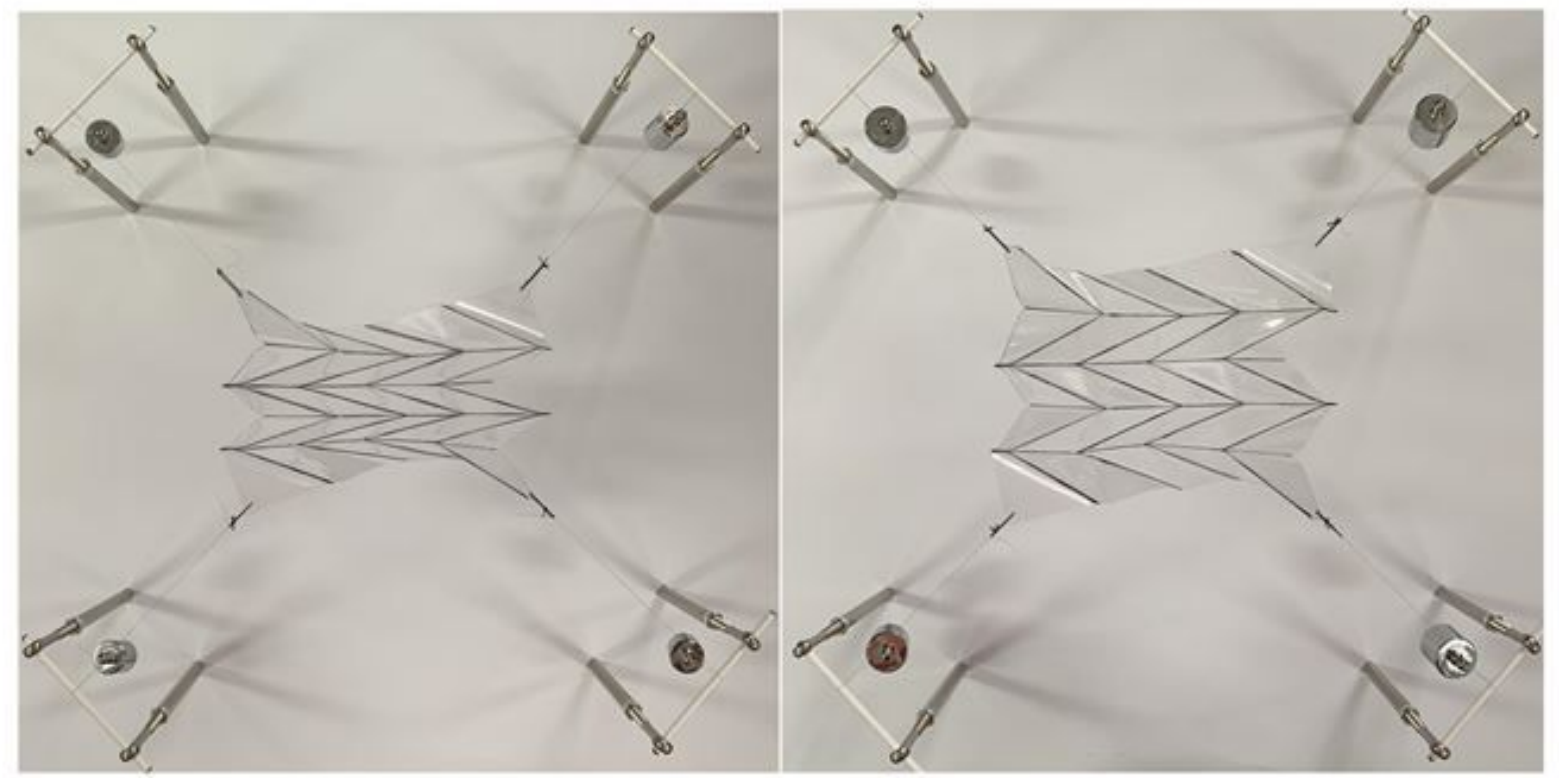

(a) $50 \mathrm{~g}(F=0.05 \mathrm{~N} / \mathrm{mm})$

(b) $100 \mathrm{~g}(F=0.1 \mathrm{~N} / \mathrm{mm})$

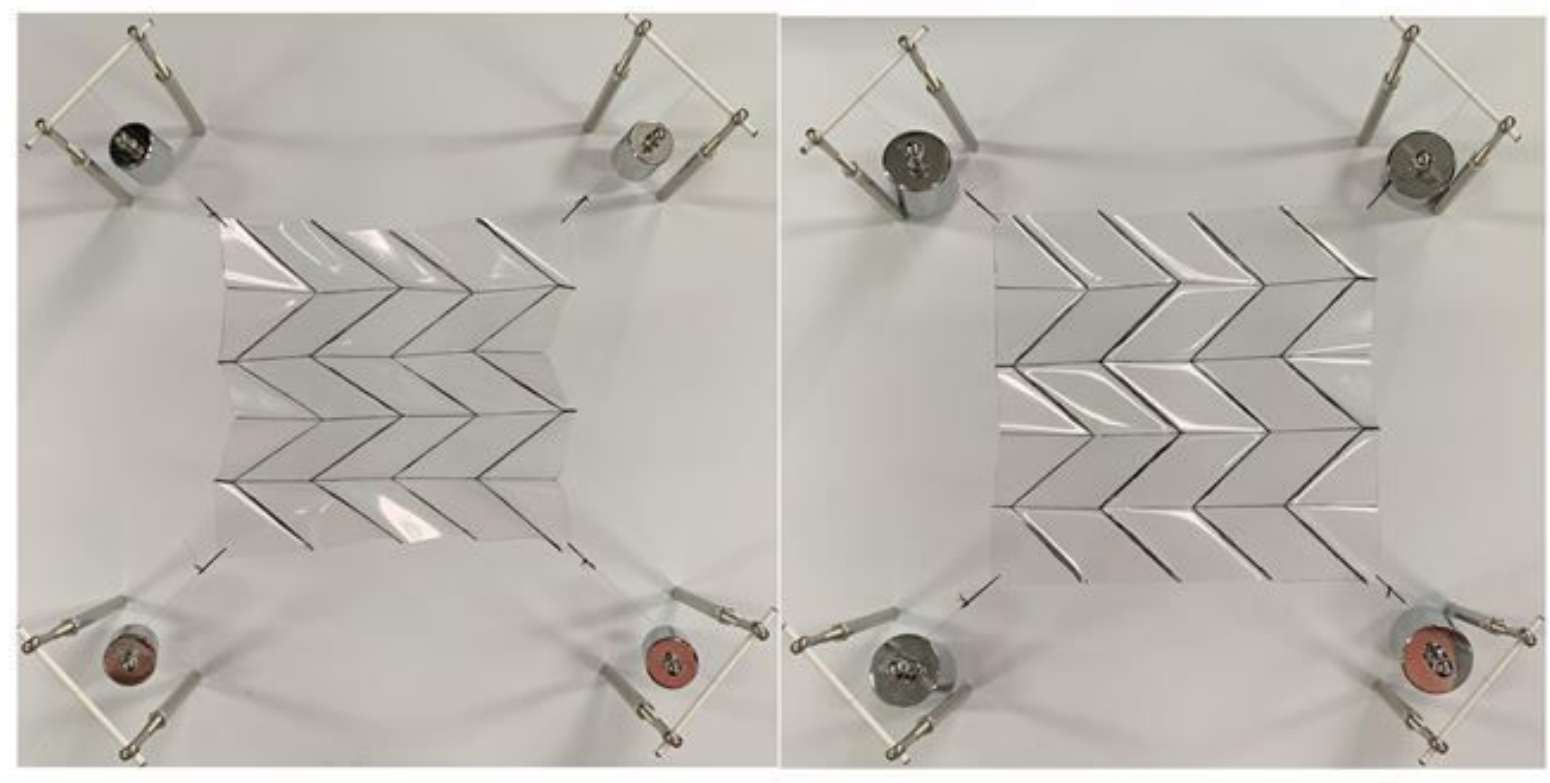
(c) $200 \mathrm{~g}(F=0.2 \mathrm{~N} / \mathrm{mm})$
(d) $500 \mathrm{~g}(F=0.5 \mathrm{~N} / \mathrm{mm})$

Figure 19

Multi-element Miura membrane deployment experiment 


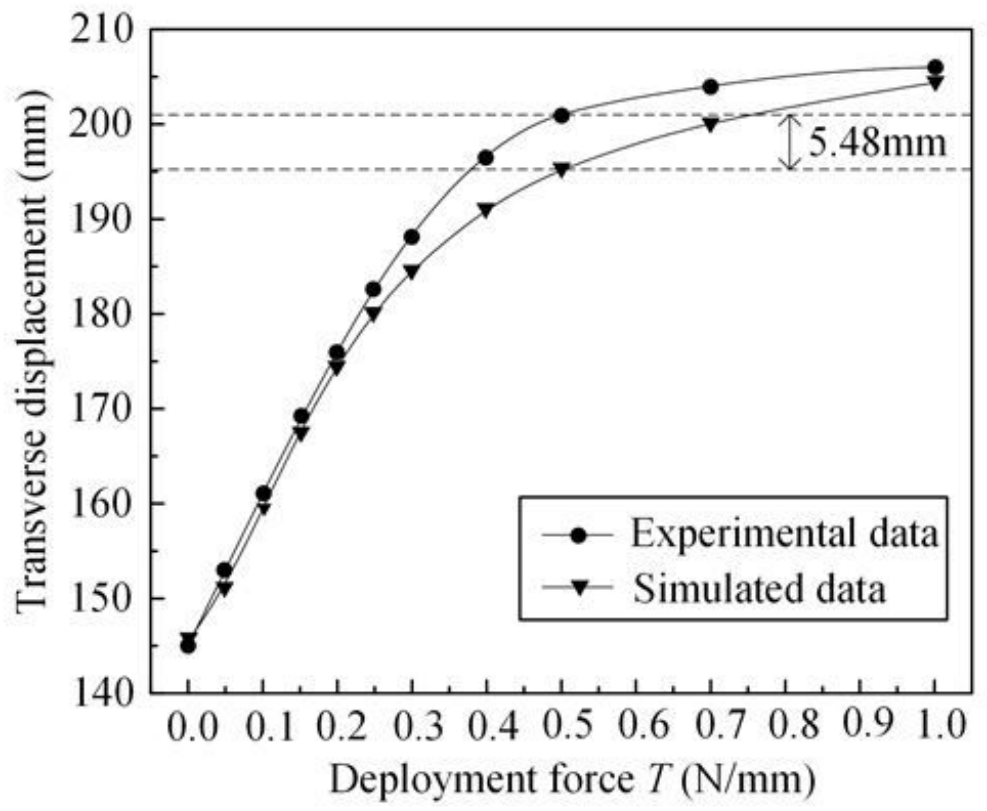

(a) The transverse displacement

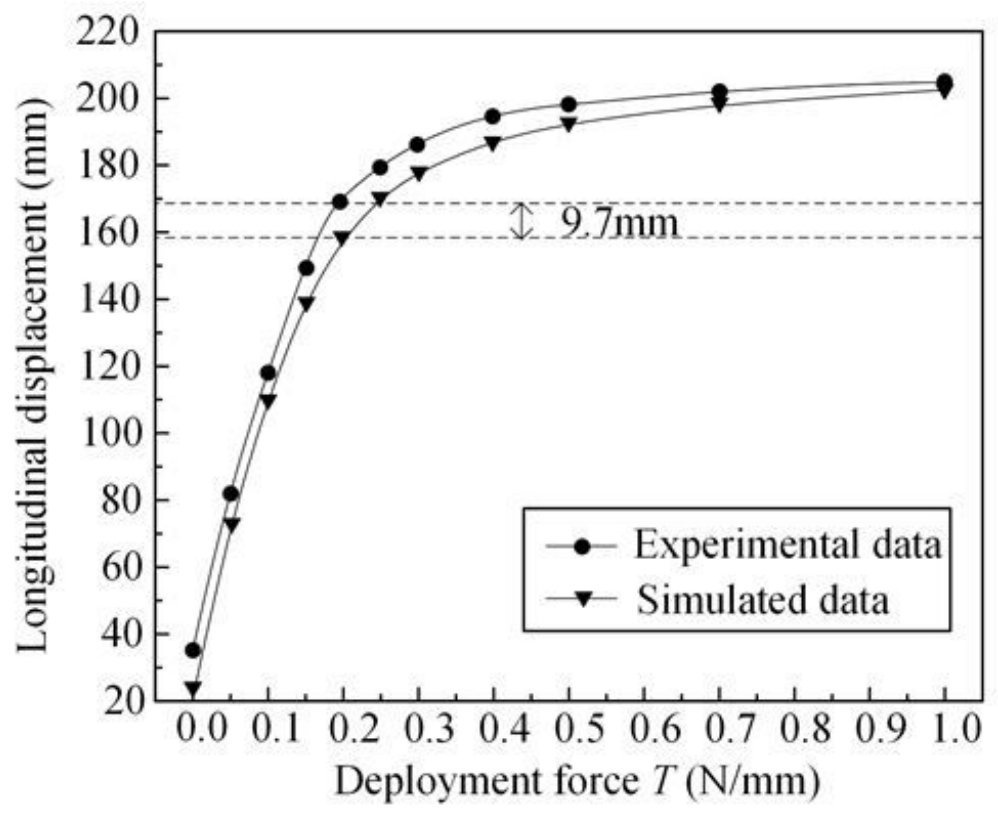

(b) The longitudinal displacement

Figure 20

Comparison of transverse and longitudinal displacements of membrane

\section{Supplementary Files}

This is a list of supplementary files associated with this preprint. Click to download. 
- declarationofcompetinginterests.docx 